\title{
Rapid dopamine transmission within the nucleus accumbens: Dramatic difference between morphine and oxycodone delivery
}

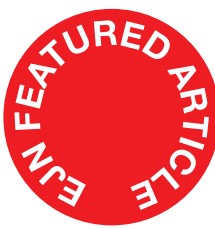

Caitlin M. Vander Weele, ${ }^{1, \dagger}$ Kirsten A. Porter-Stransky, ${ }^{1, \dagger}$ Omar S. Mabrouk, ${ }^{2}$ Vedran Lovic, ${ }^{1}$ Bryan F. Singer, ${ }^{1}$ Robert T. Kennedy ${ }^{2}$ and Brandon J. Aragona ${ }^{1,3}$

${ }^{1}$ Department of Psychology, University of Michigan, Ann Arbor, MI 48109, USA

${ }^{2}$ Departments of Chemistry and Pharmacology, University of Michigan, Ann Arbor, MI 48109, USA

${ }^{3}$ Program in Neuroscience, University of Michigan, Ann Arbor, MI, USA

Keywords: addiction, motivation, opioid, reward

\begin{abstract}
While most drugs of abuse increase dopamine neurotransmission, rapid neurochemical measurements show that different drugs evoke distinct dopamine release patterns within the nucleus accumbens. Rapid changes in dopamine concentration following psychostimulant administration have been well studied; however, such changes have never been examined following opioid delivery. Here, we provide novel measures of rapid dopamine release following intravenous infusion of two opioids, morphine and oxycodone, in drug-naïve rats using fast-scan cyclic voltammetry and rapid (1 min) microdialysis coupled with high-performance liquid chromatography - tandem mass spectrometry (HPLC-MS). In addition to measuring rapid dopamine transmission, microdialysis HPLC-MS measures changes in GABA, glutamate, monoamines, monoamine metabolites and several other neurotransmitters. Although both opioids increased dopamine release in the nucleus accumbens, their patterns of drug-evoked dopamine transmission differed dramatically. Oxycodone evoked a robust and stable increase in dopamine concentration and a robust increase in the frequency and amplitude of phasic dopamine release events. Conversely, morphine evoked a brief ( $\sim 1$ min) increase in dopamine that was coincident with a surge in GABA concentration and then both transmitters returned to baseline levels. Thus, by providing rapid measures of neurotransmission, this study reveals previously unknown differences in opioid-induced neurotransmitter signaling. Investigating these differences may be essential for understanding how these two drugs of abuse could differentially usurp motivational circuitry and powerfully influence behavior.
\end{abstract}

\section{Introduction}

Opioids, particularly $\mu$-opioid receptor (MOR) agonists, are commonly prescribed for pain management. However, opioids can cause molecular and cellular adaptations which promote physical and behavioral dependencies, and the development of addiction is therefore a major concern (Williams et al., 2001). Indeed, prescription opioid abuse has increased dramatically over the past decade (Compton \& Volkow, 2006). More recent generations of opioids (e.g. oxycodone, hydrocodone and fentanyl) are tremendously effective for pain management and, while it was initially suggested that oxycodone is unlikely to be more addictive than morphine (Davis et al., 2003), oxycodone is widely abused and is among the fastest growing drugs of abuse (Compton \& Volkow, 2006). While this is

Correspondence: Brandon J. Aragona, ${ }^{1}$ Department of Psychology, as above. E-mail: aragona@umich.edu

Robert T. Kennedy, ${ }^{2}$ Departments of Chemistry and Pharmacology, as above.

E-mail: rtkenn@umich.edu

†These authors contributed equally.

Received 25 July 2014, accepted 31 July 2014 certainly influenced by greater availability due to prescription use, recent studies suggest that oxycodone may have a higher potential to promote addiction-related behaviors than other opioids, such as morphine (Stoops et al., 2010; Comer et al., 2013). However, little is known about how oxycodone and morphine differentially influence changes in brain function associated with drug abuse and addiction.

While the mechanisms underlying drug addiction are complex and still intensely debated, dopamine (DA) neurotransmission is incontrovertibly involved in the development, maintenance and compulsive intake of abused drugs (Phillips et al., 2003a; Robinson \& Berridge, 2003; Hyman et al., 2006). Despite different mechanisms (Lüscher \& Ungless, 2006), all abused drugs increase DA transmission within the nucleus accumbens (NAc) (Di Chiara \& Imperato, 1988; Nestler \& Malenka, 2004). Techniques that allow for rapid measures of DA release have revealed that different drugs elicit distinct DA signaling dynamics, and these better inform our understanding of the neurochemical consequences of drug intake (Cheer et al., 2007; Aragona et al., 2008; Daberkow et al., 2013). Further, additional neurotransmitter systems, such as glutamate and GABA, within the NAc are critical for reward and motivation (Kel- 
ley, 2004; Wydra et al., 2013; Shen et al., 2014). Given the importance of these neurotransmitter systems in the regulation of behaviors associated with drug abuse, and that there is currently no information regarding rapid neurotransmission following opioid delivery, we aimed to determine whether rapid changes in these systems within the NAc differ following morphine or oxycodone delivery.

Given the explosive rise in prescription opioid abuse (Compton \& Volkow, 2006), the speculation that oxycodone may more potently promote drug addiction (Comer et al., 2008), and the important role for increased DA transmission in behaviors associated with drug abuse and addiction, we hypothesised that oxycodone, compared to morphine, would more robustly enhance extracellular DA concentrations ([DA]) within the NAc. Here, we employed two advanced in vivo measurement technologies, fast-scan cyclic voltammetry (FSCV) and rapid-sampling microdialysis with high-performance liquid chromatography coupled with mass spectrometry (HPLC-MS) to provide a sub-second (phasic) and minute-to-minute comparison of morphine- and oxycodone-evoked alterations in DA release. Additionally, microdialysis with HPLC-MS allowed the determination of whether these drugs differentially alter neurotransmission patterns across a wide range of signaling molecules known to be important for both modulating DA signaling and mediating reward, motivation and drug addiction.

\section{Materials and methods}

\section{Subjects}

Thirty-seven male Sprague-Dawley rats $(\sim 300 \mathrm{~g})$ were obtained with indwelling jugular vein catheters (Charles River Laboratories, Raleigh, NC, USA) and were used as subjects (FSCV morphine, $n=8$; FSCV oxycodone, $n=8$; FSCV naloxone-morphine or oxycodone, $n=9$; HPLC-MS dialysis morphine, $n=4$; HPLC-MS dialysis oxycodone, $n=5$ ). Rats were maintained on 12:12-h reverse light:dark cycle (lights off at $08.00 \mathrm{~h}$ ). Rats were housed individually with ad libitum access to food and water. All procedures were conducted in accordance with the National Institute of Health Guide for the Care and Use of Laboratory Animals and were approved by the University of Michigan Committee on the Use and Care of Animals.

\section{Surgery}

Rats were anesthetised with intramuscular ketamine hydrochloride $(90 \mathrm{mg} / \mathrm{kg})$ and xylazine hydrochloride $(10 \mathrm{mg} / \mathrm{kg})$. For FSCV subjects, a guide cannula (Bioanalytical Systems, West Lafayette, IN, USA) was secured dorsal to the NAc core (AP, +1.3; ML, \pm 1.3 ; $\mathrm{DV},-2.5 \mathrm{~mm}$ relative to bregma) or shell (AP, +1.8 ; ML, \pm 0.8 ; $\mathrm{DV},-2.5 \mathrm{~mm})$ and a $\mathrm{Ag} / \mathrm{AgCl}$ reference electrode was placed in the contralateral cortex $(\mathrm{AP},-0.8 ; \mathrm{ML}, \pm 3.0 ; \mathrm{DV},-2.5 \mathrm{~mm}$ relative to bregma). A bipolar stimulating electrode (Plastics One, Roanoke, VA, USA) was lowered into the ventral tegmental area (VTA; AP, -5.2 ; ML, $\pm 0.8 \mathrm{~mm}$ relative to bregma) until electrically-evoked DA release within the striatum was detected, as described previously (Wightman et al., 2007). Similarly, microdialysis subjects were implanted with a guide cannula (CMA, Holliston, MA, USA) positioned $1 \mathrm{~mm}$ above the NAc (AP, +1.7; ML, \pm 0.9 ; DV, $-6.0 \mathrm{~mm}$ ). All implants were permanently secured to the skull with stainless steel surgical screws and dental acrylic. Following surgery, rats recovered for $\sim 5$ days until they reached pre-operative weight.

\section{Experimental procedures: FSCV}

On the day of the pharmacological experiment, a recording cable was anchored to the skull cap and connected to a rotating commutator (Crist Instruments, Hagerstown, MD, USA) suspended from the top of the test chamber allowing rats to move freely about the Plexiglas recording chamber. Vehicle-loaded tubes were threaded through the rotating commutator and along the headstage cable, and attached to the i.v. catheter and a syringe pump outside of the test chamber. Carbon-fiber microelectrodes $(\sim 120 \mu \mathrm{m}$ in length) were lowered into the NAc core or shell and secured in locations where both electrically-evoked and naturally occurring phasic DA release events were detected (Wightman et al., 2007).

Similar to our previous FSCV studies (Aragona et al., 2008; Porter-Stransky et al., 2011), experiments began with a 15-min baseline recording interval followed by another 15 -min recording that controlled for i.v. vehicle administration $(0.2 \mathrm{~mL}$ of sterile saline; $5 \mathrm{~s}$ ). Rats were then administered, at 15-min intervals, cumulative doses of morphine sulfate (Sigma, St Louis, MO, USA) or oxycodone hydrochloride (Sigma) dissolved in sterile saline $(0.2 \mathrm{~mL} ; 5 \mathrm{~s})$. Following saline injections, separate groups of animals received infusions of either oxycodone or morphine according to the following doses: low dose, $0.1 \mathrm{mg} / \mathrm{kg}$; medium dose, $0.5 \mathrm{mg} / \mathrm{kg}$; and high dose, $1.0 \mathrm{mg} / \mathrm{kg}$ i.v. Drug doses were chosen based on what rats selfadminister with the lowest dose under morphine self-administration thresholds (Mierzejewski et al., 2003). The clinical morphine-tooxycodone potency ratio has been shown to be between $1: 1$ and $1: 1.5$, with large individual differences in bioavailability (Davis et al., 2003). As such, oxycodone doses were kept equivalent to morphine doses.

Another control, using the MOR-preferring antagonist naloxone, was conducted to test the involvement of MORs in the opioidevoked increases in DA transmission identified in the present study. A separate group of rats received baseline and saline recording periods followed by a pre-exposure of naloxone, $3.0 \mathrm{mg} / \mathrm{kg}$, i.v. (Tocris, Bristol, UK). Fifteen minutes after the naloxone infusion, either morphine or oxycodone $(0.5 \mathrm{mg} / \mathrm{kg})$ was administered because this dose consistently elicited a similarly initial increase in [DA]. For all subjects, the final recording session was followed by administration of a DA autoreceptor blocker, the D2-type receptor antagonist raclopride (Sigma; $1.0 \mathrm{mg} / \mathrm{kg}$ i.v.; $5 \mathrm{~s}$ ) as a positive control. This dose of raclopride blocks somatodendritic and terminal autoreceptors (Andersson et al., 1995) and robustly increases DA transients and [DA] (Aragona et al., 2008).

\section{FSCV data acquisition}

Carbon-fiber microelectrodes were lowered into the NAc core or shell and a negative holding potential of $-0.4 \mathrm{~V}$ was applied. Then, at $10 \mathrm{~Hz}$, a triangular voltage ramp was applied (Fig. 1A; oxidative scan, -0.4 to $1.3 \mathrm{~V}$; reductive scan, 1.3 to $-0.4 \mathrm{~V}$ at a rate of $400 \mathrm{~V} / \mathrm{s}$ ); this results in a large and highly consistent change in current that is recorded from the carbon surface (Robinson et al., 2003) (Fig. 1A, insets i-iv). As the charging currents resulting from these voltage ramps are stable (Robinson et al., 2003), they are continually background-subtracted in order to examine the changes in current associated with changes in concentration of electroactive species surrounding the carbon fiber (Fig. 1B-D) (Robinson et al., 2003). As such, FSCV data are presented as a delta, and background-subtracted data are the norm for measuring phasic changes in neurotransmission, including DA release (Garris \& Wightman, 1994; Phillips et al., 2003b; Willuhn et al., 2012). Current changes 
A

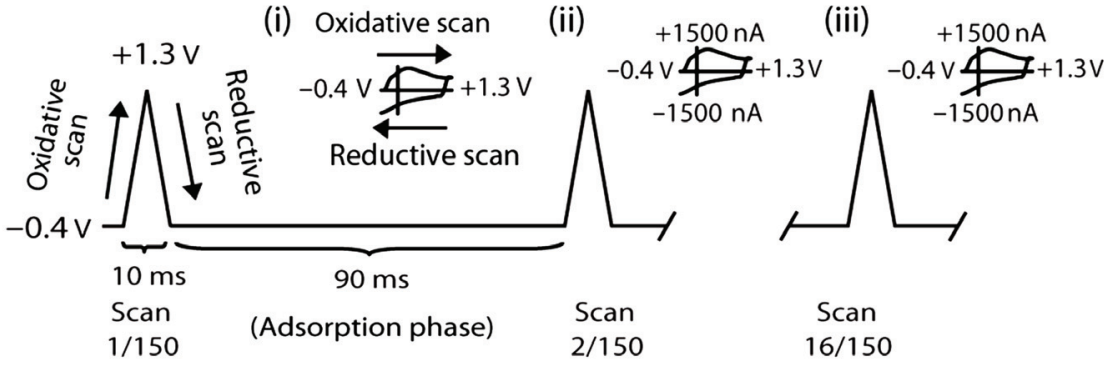

B
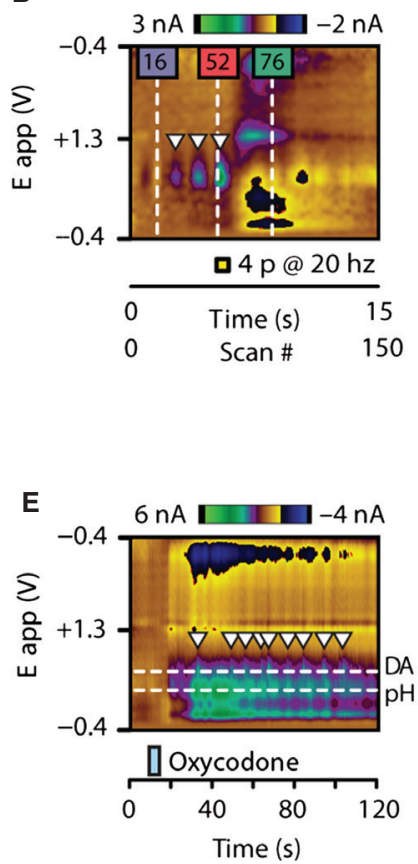

C Peak DA

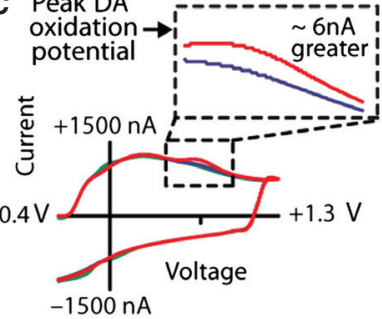

$-1500 \mathrm{nA}$
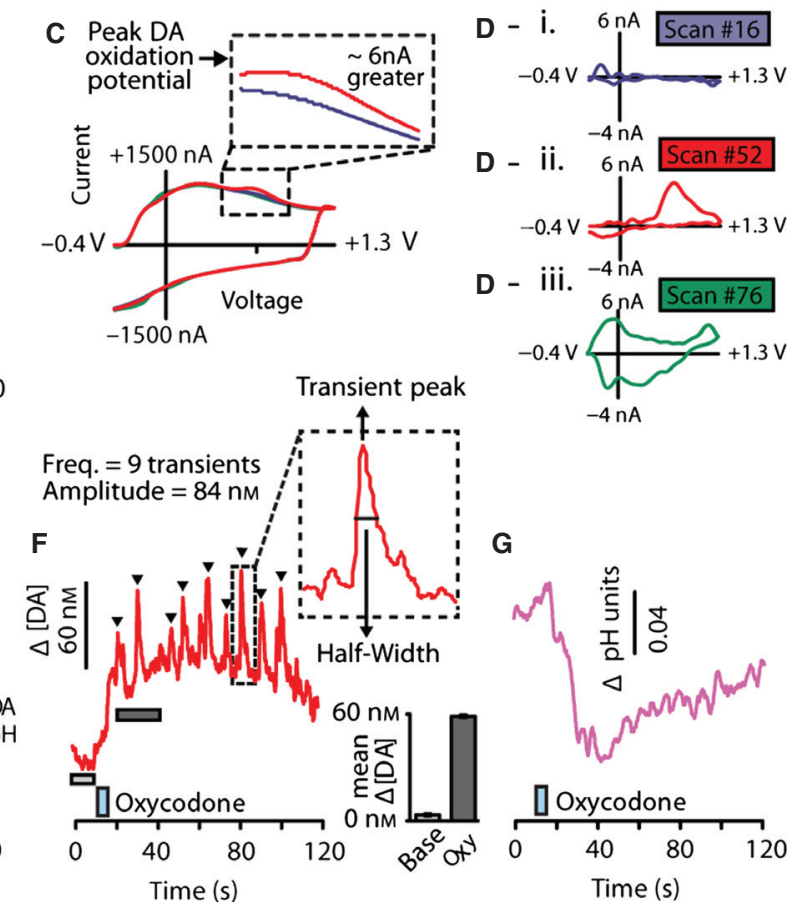

FIG. 1. Measuring real-time neurochemical transmission dynamics with background-subtracted FSCV. (A) The carbon-fiber microelectrode is held at -0.4 V for $90 \mathrm{~ms}$ between voltage ramps. This 'adsorption phase' attracts positive electroactive analytes because of the negative potential applied and causes them to adsorb onto the carbon surface. At a rate of $10 \mathrm{~Hz}$, the holding potential is rapidly ramped (400 V/s) to a positive voltage (1.3 V; 'oxidative scan') and then back down to the negative holding potential $(-0.4 \mathrm{~V}$; 'reductive scan'). This triangular scan produces a highly robust increase in current at the carbon surface, called the 'charging current' (insets i to iii in A). Each scan (which has a corresponding CV; see below) is represented along the $x$-axis [150 measures $(150 \mathrm{CVs})$ in $15 \mathrm{~s}$. Highlighted are several representative scans and the resulting current changes which they cause. (B) Background-subtracted changes in current measured during the triangular ramp are plotted in false color across the change in voltage associated with the ramp ( -0.4 to $1.3 \mathrm{~V}$, then back to $-0.4 \mathrm{~V}$; plotted on a straight line along the $y$-axis). (C) Each voltage ramp generates a robust charging current. The charging current is extremely stable which allows for FSCV data to be background-subtracted. This process permits acute current surges beyond that of the charging current to be detected. (D) Unique CVs from three different ramps (from scans 16, 52 and 76) are shown (i to iii respectively). These demonstrate how small the acute changes in current caused by changes in neurochemical concentration are relative to the magnitude of the charging current (as the inset in Fig 1C shows, the red scan is slightly larger than the background scan). CVs from the three selected ramps; the approximate difference in current is shown for each CV (16, 52 and 76). The blue scan (D i) shows a typical charging current, after background subtraction, (taken from a location in the color plot where there was not an obvious change in color). Conversely, the red scan (D ii) shows the current at scan 52 which is $200 \mathrm{~ms}$ after the DA neurons in the VTA received electrical stimulation (yellow box). The CV reveals an increase in current caused by an increase in DA concentration at the recording site. Electrical stimulation also elicited an expected delayed decrease in current attributed to a basic shift in $\mathrm{pH}$. The $\mathrm{CV}$ for this is shown by scan 76 in D iii. (E) Color plot resulting from a representative infusion of oxycodone (blue box). Two reliable neurochemical events are evoked by opioid infusion, an increase in DA concentration and an acidic shift in pH. (F) [DA] trace (red), which corresponds to the upper vertical dotted line in Fig 1E, and DA transients are clearly visible (triangles). Mean [DA] change (bar graph inset) can be determined by averaging a period of time prior to the infusion (light gray bar) and a time period after the infusion (dark gray bar). The inset shows that further transient analysis (amplitude and half-width) provides further resolution underpinning DA transmission. (G) Phasic changes in acidic pH following oxycodone delivery (blue box).

are detected at the surface of the carbon-fiber microelectrodes, and changes beyond those caused by the charging current are plotted in false color against time and the applied voltage ramp (Fig. 1B) (Michael et al., 1999). This allows convenient visualisation of neurochemical changes across the entire applied voltage ramp. With this particular optimisation, FSCV robustly detects changes in [DA] as well as changes in local pH (Fig. 1D and E) (Venton et al., 2003; Roitman et al., 2004; Takmakov et al., 2010; Badrinarayan et al., 2012). Here, we show current changes measured at the carbon fiber during electrical stimulation of DA neurons with and without background subtraction (Fig. 1C) and one can easily see that the DA released by the stimulation causes a increase in current beyond that produced by the charging voltage of the applied potential needed to measure phasic DA with FSCV.

Specific chemical species are identified by their characteristic change in current and this is identified by the shape of its cyclic voltammogram (CV; Fig. 1D) (Robinson et al., 2003; Heien et al., 2005). CVs are current-voltage traces that provide electrochemical 
'fingerprints' that identify each electroactive species because of their unique oxidation and reduction reactions during the voltage ramp (Phillips et al., 2003a). Following background subtraction, as minimal changes in current occurred before the representative electrical stimulation shown, the CV just prior to the stimulation shows very low changes in current (Fig. 1, D-i, scan 16). However, electrical stimulation of the VTA caused a rapid increase in current at the peak oxidation potential for DA (Fig. 1B and D-ii, scan 52). As reliably shown in previous studies, electrical stimulation of DA neurons was also followed by a slow and long-lasting change in basic $\mathrm{pH}$ (Fig. 1E and G) compared to evoked DA. To convert current to [DA] and $\mathrm{pH}$ units, electrodes were calibrated in a microfluidic flow system (Sinkala et al., 2012) with solutions of known DA concentration and differing $\mathrm{pH}$ values to generate calibration curves (Badrinarayan et al., 2012). Recorded CVs were converted from current into [DA] using principal component analyses using electricallyevoked DA and $\mathrm{pH}$ CVs acquired before and after each experiment as training sets for principal component analysis (Heien et al., 2005; Keithley et al., 2009; Keithley \& Wightman, 2011). This allows the separation of DA from $\mathrm{pH}$ and the conversion of current changes into nM concentations for DA and $\mathrm{pH}$ units (Heien et al., 2005; Keithley et al., 2009; Keithley et al., 2010). Electrical stimulations of the VTA were used to optimally place FSCV electrodes within the NAc, to verify electrode fidelity throughout the experiment, and to generate training sets.

\section{FSCV analysis}

As in our previous experiments (Porter-Stransky et al., 2011; Badrinarayan et al., 2012), carbon-fiber electrodes were constructed in all aspects as described previously (Robinson et al., 2003) except that epoxy was applied to the seal where the carbon meets the glass encasement. Waveform generation, data collection, filtering and analysis were performed as previously described (Wightman et al., 2007). A DA transient was defined as a five-fold or greater phasic surge in [DA], relative to the root-mean-square noise, that was $\sim 1 \mathrm{~s}$ in duration (Fig. 1F) (Phillips et al., 2003a). Data were collected in 30-s files and background-subtracted at approximately the lowest current value. Current was converted to [DA] using principal component analysis (Keithley \& Wightman, 2011), and transient detection and quantification were achieved using MATLAB (code provided by Dr Richard Keithley) and MINIANALYSIS (Synaptosoft, Decatur, GA, USA), respectively. As established in previous studies (Aragona et al., 2008, 2009; Badrinarayan et al., 2012), transients $<20 \mathrm{~nm}$ were not included in the analysis because events below this magnitude are not reliably detected across electrodes.

\section{Microdialysis with HPLC-MS}

As the FSCV studies used here are highly optimised to measure changes in DA and $\mathrm{pH}$, we employed rapid microdialysis coupled with a novel HPLC-MS method (Song et al., 2012) to measure 1 -min changes in [DA], as well as changes in 15 additional neurochemical species, with high sensitivity. Dialysate samples were collected and prepared as previously described (Song et al., 2012). Briefly, at the beginning of microdialysis experiments, a 1-mm probe was inserted into a guide cannula to target the NAc. Microdialysis probes were perfused at $2 \mu \mathrm{L} / \mathrm{min}$ for $1.5 \mathrm{~h}$ prior to sample collection using a Fusion 400 syringe pump (Chemyx, Stafford, TX, USA). Samples were then collected every $60 \mathrm{~s}$, yielding $2-\mu \mathrm{L}$ samples. Immediately after dialysate collection, the following reagents were added to each $2.0-\mu \mathrm{L}$ dialysate fraction: $1.5 \mu \mathrm{L}$ of borate buffer (sodium tetraborate, $100 \mathrm{~mm}$ ), $1.5 \mu \mathrm{L}$ benzoyl chloride in $2 \%$ acetonitrile, $1.5 \mu \mathrm{L}$ of stable-isotope labeled internal standard solution, and $1.5 \mu \mathrm{L}$ d4-acetylcholine (ACh) internal standard solution (Song et al., 2012) (Fig. 9A). Resulting fractions were analysed using a nanoAcquity HPLC system (Waters, Milford, MA, USA) equipped with a Waters $1 \times 100 \mathrm{~mm}$ HSS T3 reverse-phase HPLC column operated at $100 \mu \mathrm{L} / \mathrm{min}$. Eluting analytes were detected using an Agilent 6410 triple quadrupole MS (Agilent, Santa Clara, CA, USA) operating in positive mode performing dynamic multiple-reaction monitoring. The following compounds were monitored in dialysate by the HPLC-MS method: DA and its metabolites 3-methoxytyramine (3-MT), homovanillic acid (HVA) and 3,4-dihydroxyphenylacetic acid (DOPAC); norepinephrine and its metabolite normetanephrine; serotonin and its metabolite 5-hydroxyindole-3-acetic acid; adenosine; histamine; aspartate; serine; taurine; glutamate; and GABA (Fig. 9A and B). $\mathrm{ACh}$, which cannot be labeled with benzoyl chloride, was detected directly.

We collected 15 baseline samples and five samples following an i.v. saline infusion. Next, subjects received an i.v. infusion $(200 \mu \mathrm{L})$ of $0.5 \mathrm{mg} / \mathrm{kg}$ of either morphine or oxycodone, and fractions were collected for $15 \mathrm{~min}$. These doses were chosen because FSCV studies revealed that these doses evoked similar magnitudes in peak increases in [DA] evoked by drug infusion and caused similar durations in behavioral immobility (data not shown). As cocaine in the presence of an autoreceptor blocker (the D2-type antagonist raclopride) has been shown to synergistically increase [DA] (Rouge-Pont et al., 2002; Aragona et al., 2008), co-infusions of raclopride and cocaine were administered as a positive control in a subset of subjects. Specifically, $1 \mathrm{mg} / \mathrm{kg}$, i.v. raclopride was administered at the end of the experiment and was followed $10 \mathrm{~min}$ later by $3 \mathrm{mg} / \mathrm{kg}$, i.v. cocaine infusion. Dialysate was collected for $10 \mathrm{~min}$ following these infusions.

\section{Statistics}

To compare mean changes from pre-infusion basal levels in [DA] measured by FSCV across all drug doses, 120-s sampling periods were averaged into $10-\mathrm{s}$ or 1 -s bins and a linear mixed model was utilised because of its ability to handle repeated-measures data in which observations are not independent (Aragona et al., 2008). Comparisons of transient frequency, amplitude and halfwidth following drug treatment compared to mean saline values were also determined using linear mixed-model regressions (data binned into 1-min bins for the duration of the 15-min recording period). Potential core-shell differences were assessed using a liner mixed model by including the region as a fixed-effect variable. To compare mean changes in DA transients across entire recording sessions, transient frequency, amplitude and half-width per min across drug doses were compared to control infusions of saline using one-way ANOvas with Bonferroni post-test corrections for multiple comparisons. For microdialysis data, neurotransmitter concentrations were normalised and converted to percentages of baseline. To statistically determine drug-induced changes in neurotransmitter and metabolite levels, a linear mixed model was utilised. The linear mixed models calculated specific $t$ - and $P$-values for each time point as well as conducting an omnibus $F$-test. $F$ values are reported to examine overall main effects and $t$-values are reported to assess differences for specific time points and are corrected for multiple comparisons. The significance of each time point was determined when calculated $t$-values crossed the thresh- 
old of the critical $t$-value, which corresponded to an $\alpha$ level of 0.05. Degrees of freedom were adjusted based on the distance between comparisons in this model. All statistical analyses were conducted using SPSS version 19 for Windows, and graphs were generated in Graph Pad Prism and Adobe Illustrator. All data are presented as mean \pm SEM and an $\alpha$ level of 0.05 was used for determining statistical significance $(*)$ for all analyses. An $\alpha$ level of 0.09 was used to indicate trending significance (\#).

\section{Histology}

Following experiments, carbon-fiber FSCV electrodes were removed and rats were killed with ketamine $(0.2 \mathrm{~mL}$ i.v.). In FSCV subjects, an electrolytic lesion was made to mark the location of the recording electrode using a tungsten electrode at the location of the recording site through the guide cannula at the microdrive setting used during testing (Aragona et al., 2008). Probe placement for microdialysis studies could be determined easily with light microscopy upon removal of the probe, using the same histological preparations. Brains were removed and post-fixed in formalin. Brains were sectioned into 40 - to $50-\mu \mathrm{m}$ coronal slices on a cryostat and stained with Cresyl violet to verify electrode placement.
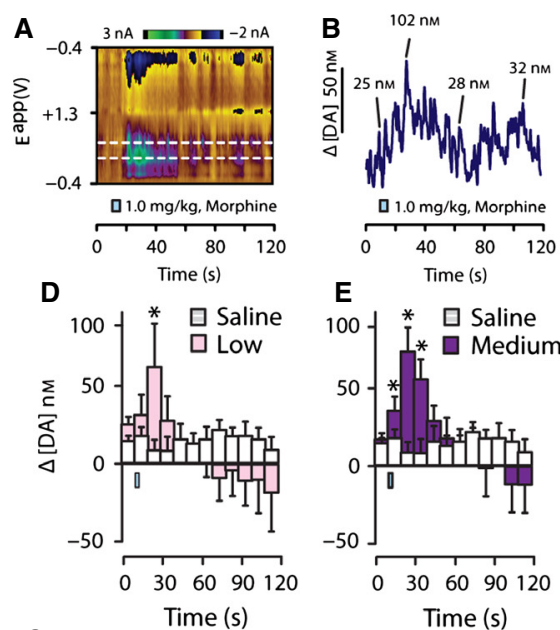

\section{C}
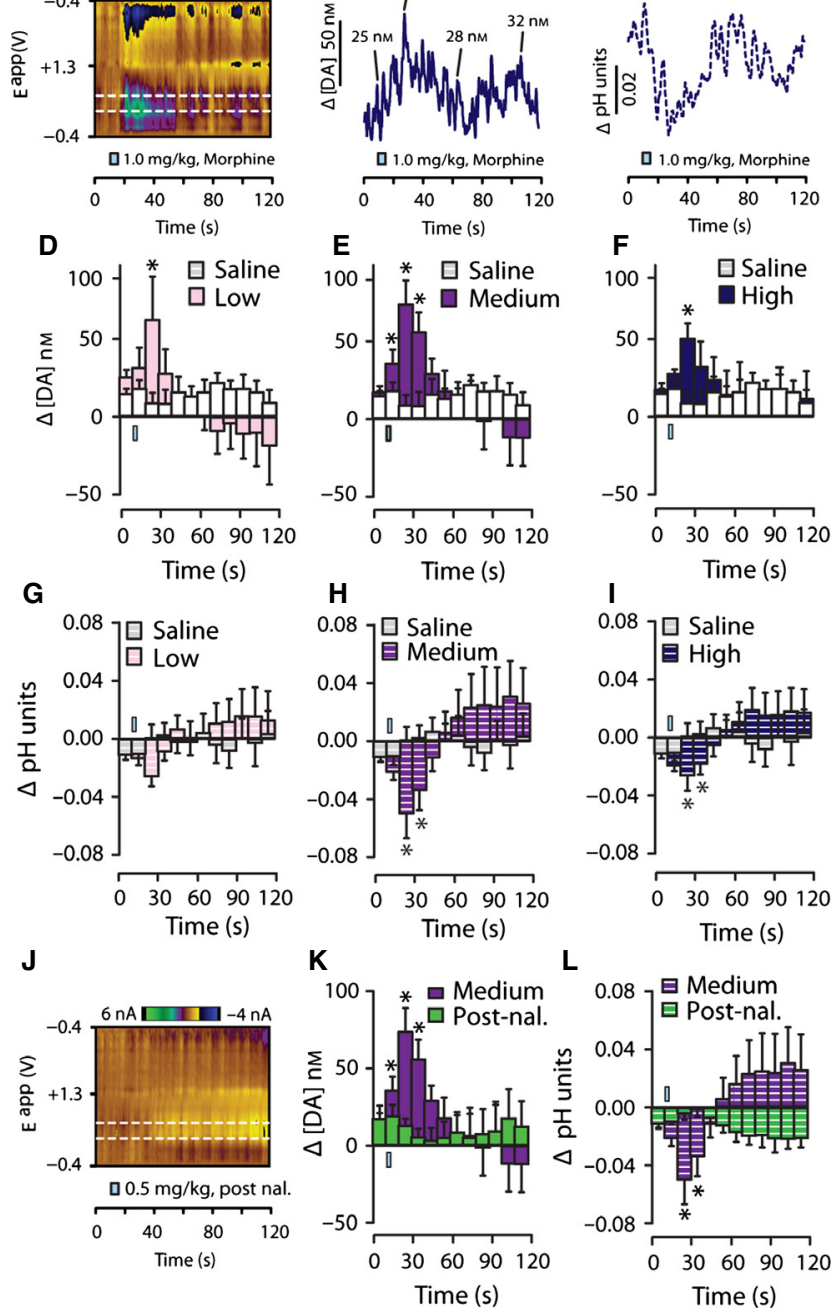

\section{Results}

\section{Dramatic differences in dopamine concentration evoked by morphine and oxycodone}

Detection of naturally-occurring transients is necessary for accurate FSCV measurements of pharmacologically-induced changes in DA transmission (Wightman et al., 2007); therefore, carbon-fiber electrodes were lowered into the NAc core or shell to locations where both electrically-stimulated DA release and naturally-occurring phasic release occurs (i.e. DA transients). A representative color plot (Fig. 2A) and corresponding traces (Fig. 2B and C) demonstrate that i.v. infusion of morphine resulted in a rapid increase in [DA] (Fig. 2B) and an acidic shift in pH (Fig. 2C).

To quantify these data, [DA] was binned every $10 \mathrm{~s}$ and drugevoked changes were compared to those following control infusions of saline. The low dose $(0.1 \mathrm{mg} / \mathrm{kg})$ infusion of morphine significantly elevated [DA] only within the first bin immediately following drug infusion (Fig. $2 \mathrm{D} ; t_{79.149}=2.133, P=0.036$ ). Following the medium dose $(0.5 \mathrm{mg} / \mathrm{kg})$, morphine evoked significant increases in [DA] for the first $\left(t_{76.088}=2.491, \quad P=0.015\right)$, second $\left(t_{81.698}=5.591, P<0.001\right)$ and third $\left(t_{83.963}=3.229, P=0.002\right)$ bins following drug infusion (Fig. 2E). As with the low dose, the high dose $(1.0 \mathrm{mg} / \mathrm{kg})$ of morphine evoked increases in [DA] only within the second bin following drug infusion (Fig. 2F; $\left.t_{69.705}=3.587, P=0.001\right)$.

To test whether these increases in DA release were mediated by activation of MORs, we conducted an additional experiment in which subjects were pretreated with the MOR antagonist naloxone $(3.0 \mathrm{mg} / \mathrm{kg}$ ) prior to morphine infusion. For this test, the medium

FIG. 2. Average acute changes in dopamine concentration and local $\mathrm{pH}$ in the NAc evoked by i.v. morphine. (A) A representative color plot shows increased DA transmission (top white line) and an acidic shift in local $\mathrm{pH}$ (bottom white line) following i.v. administration of the high dose of morphine $(1.0 \mathrm{mg} / \mathrm{kg})$. (B) Current was converted into [DA] and trace is representative of the peak DA oxidation potential. Select DA transients were identified and their respective amplitudes are given. (C) Similarly, current from the representative color plot was converted into changes in $\mathrm{pH}$ units and the $\mathrm{pH}$ trace was taken from the lower dotted white line on the color plot. (D-I) Change in [DA] and $\mathrm{pH}$ units were binned every $10 \mathrm{~s}$ across a 120 -s sampling window for statistical comparison to pre-infusion values. (D-F) Changes in [DA] during infusions of cumulatively increasing doses of morphine compared to control infusions of saline. (D) Following the low morphine dose $(0.1 \mathrm{mg} / \mathrm{kg}$, i.v. $)$, [DA] was increased in the second time bin. (E) Following the medium morphine dose $(0.5 \mathrm{mg} / \mathrm{kg}$, i.v.) [DA] was increased in the first, second and third time bins. (F) As with the low dose, following the high morphine dose $(1.0 \mathrm{mg} / \mathrm{kg}$, i.v.) [DA] was only increased during the second time bin. (G-I) Changes in $\mathrm{pH}$ units following infusions of cumulatively increasing doses of morphine compared to infusions of saline. (G) The low dose of morphine did not significantly alter $\mathrm{pH}$ units. $(\mathrm{H})$ Following the medium dose of morphine there was a significant acidic shift in $\mathrm{pH}$ during the first, second and third time bins following morphine infusion. (I) Additionally, high dose morphine infusion also caused an acidic shift in local $\mathrm{pH}$ during the first, second and third time bins. (J-L) To examine whether MORs were dependent on the rapid neurochemical changes following morphine delivery, the most effective dose of morphine was chosen $(0.5 \mathrm{mg} / \mathrm{kg})$ to examine rapid changes in [DA] and $\mathrm{pH}$ following blockade of MORs via pre-treatment with the MOR-preferring antagonist naloxone (3.0 $\mathrm{mg} / \mathrm{kg}$, i.v.; given $15 \mathrm{~min}$ prior to morphine infusion). (J) A representative color plot demonstrating that following naloxone pre-treatment, morphine-evoked increases in DA transmission and the acidic shift in $\mathrm{pH}$ were eliminated. (K) Quantitatively, while the medium dose of morphine $(0.5 \mathrm{mg} / \mathrm{kg})$ significantly increased [DA] following a saline pre-treatment, this effect was abolished if morphine infusion was preceded by naloxone. (L) Naloxone pretreatment also abolished morphine-evoked changes in $\mathrm{pH}$. Error bars indicate SEM; $* P<0.05$. The i.v. infusion is indicated by a blue rectangle which is accurate according to the time and duration of saline or drug infusion. 
dose of morphine (i.e., the dose that evoked the most robust effects, $0.5 \mathrm{mg} / \mathrm{kg}$ ) was used. Administration of the MOR-preferring antagonist naloxone eliminated the morphine-evoked increases in [DA] as demonstrated in the color plot (Fig. 2J) as well as quantification of the data using the same 10 -s binning (Fig. $2 \mathrm{~K} ; F_{11,53.572}=1.264$, $P=0.270)$.

In addition to measuring real-time DA changes, these voltammetric recording conditions also measure local changes in $\mathrm{pH}$ (see Fig. 1E and G) (Takmakov et al., 2010; Badrinarayan et al., 2012). Our FSCV studies show that opioids, including oxycodone (below), morphine and remifentanil (Vander Weele et al., 2011), all evoke an acidification in local $\mathrm{pH}$. This novel observation may reflect an increase in carbon dioxide blood concentration due to opioidinduced respiratory depression (Thompson et al., 1995; Takmakov et al., 2010). The low dose drug infusion did not alter pH (Fig. 2G; $P>0.05$ at all time points). However, following both the medium and high doses, morphine caused significant acidification during the second and third time bin following drug infusion (Fig. $2 \mathrm{H}$, medium dose, $t_{81.234}=-3.681, P<0.001$; and Fig. $1 \mathrm{I}, t_{81.416}=-2.137$, $P=0.001)$. Similar to its effects on DA, pre-exposure to naloxone eliminated acidic shifts in $\mathrm{pH}$ (Fig. $2 \mathrm{~J}$ and $\mathrm{L}, F_{11,36.642}=0.950$, $P=0.506)$.

In contrast to morphine, oxycodone caused more robust acute changes in $[\mathrm{DA}]$ and acidic shifts in $\mathrm{pH}$, as can be seen by inspection
A

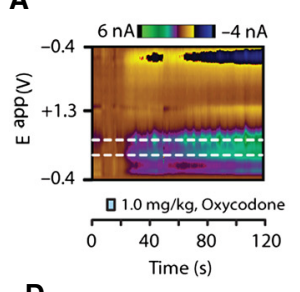

D
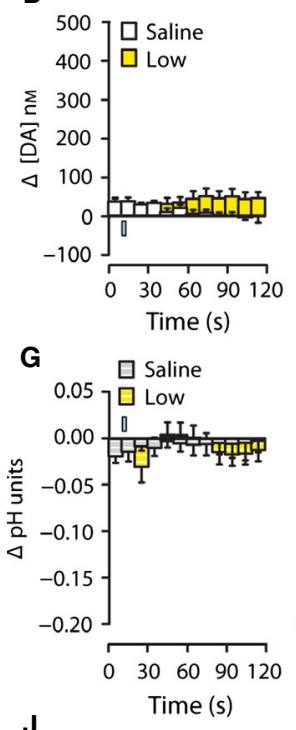

$\mathbf{J}$

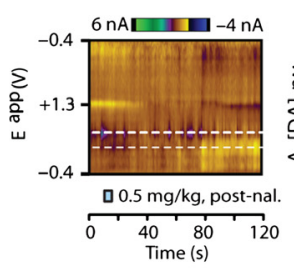

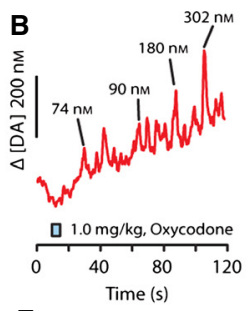

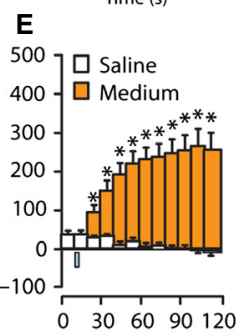

Time (s)
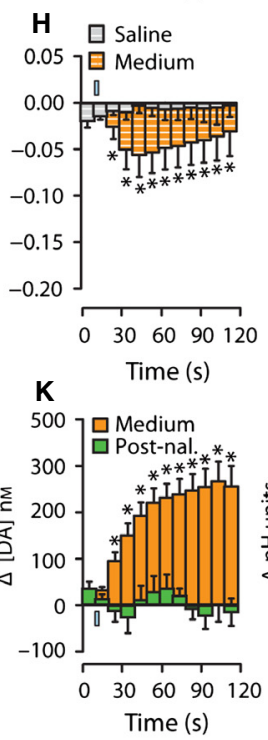

C
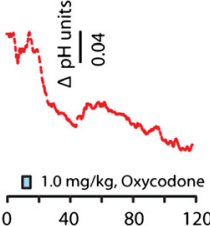

$\mathbf{F}$

5007 Saline
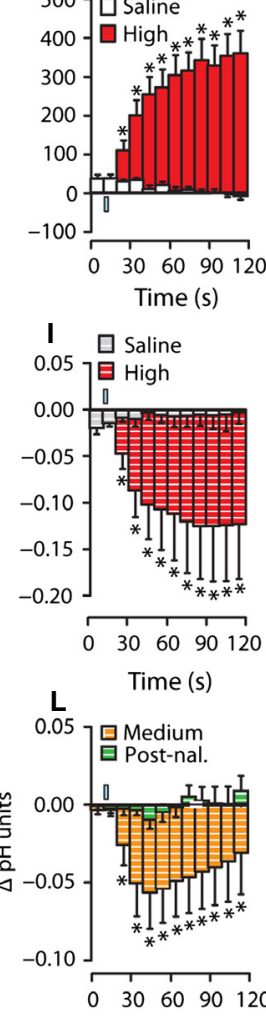

Time (s) of the representative color plot and corresponding traces (Fig. 3A-C). The dose of oxycodone that evoked the greatest response $(1 \mathrm{mg} / \mathrm{kg})$ caused a dramatic increase in [DA], composed of both a gradual rise in concentration and a robust increase in DA transients superimposed upon this rise (Fig. 3B). These representative examples also show robust $\mathrm{pH}$ acidification (Fig. 3C). Unlike morphine, responses following oxycodone were highly dose-dependent. Low dose administration of oxycodone did not significantly elevate [DA] (Fig. 3D; $F_{11,73.847}=1.495, P=0.152$ ). However, both medium (Fig. 3E; $F_{11,75.752}=14.653, \quad P<0.001$ ) and high (Fig. 3F; $F_{11,75.752}$ $=14.653, P<0.001)$ doses of oxycodone significantly increased [DA] in the second time bin following drug infusion, and [DA] continued to rise throughout the recording period (Fig. 3E and F).

Similar to its effects on DA, the low dose infusion of oxycodone did not alter $\mathrm{pH}$ (Fig. 3G; $P>0.05$ all time points). However, both medium and high doses of oxycodone caused robust acidic $\mathrm{pH}$ shifts beginning at the second recording bin and lasting throughout the recording period (Fig. $3 \mathrm{H}$ and I; $P<0.05$ at the aforementioned time points). As with morphine, naloxone pre-treatment eliminated oxycodone-induced increases in [DA] and acidic shifts in $\mathrm{pH}$, evident from the representative color plot (Fig. 3J) and time-averaged data (Fig. $3 \mathrm{~K}$ and $\mathrm{L} ; F_{11,20.194}=1.615, P=0.169$ ).

\section{Morphine and oxycodone differentially alter phasic dopamine release events}

Thus far we have shown that morphine and oxycodone caused very different changes in [DA] immediately following drug infu-

FIG. 3. Average acute changes in dopamine concentration and local $\mathrm{pH}$ in the NAc evoked by i.v. oxycodone. (A) A representative color plot shows increased DA transmission and an acidic shift in local $\mathrm{pH}$ following i.v. administration of the high dose of oxycodone $(1.0 \mathrm{mg} / \mathrm{kg})$. (B) Current was converted into $[\mathrm{DA}]$ and the trace shown represents data gathered from the peak DA oxidation potential (see top white dotted line in Fig. 6A). Select DA transients are identified and their respective amplitudes given. (C) Similarly, current from this color plot was converted into changes in $\mathrm{pH}$ units and the corresponding trace (taken from the lower dotted white line in Fig. 6A) is shown. (D-I) [DA] and $\mathrm{pH}$ were binned every $10 \mathrm{~s}$ across the 120-s sampling window for comparison to pre-infusion values. (D-F) Changes in [DA] during infusions of cumulatively increasing doses of oxycodone compared to control infusions of saline. Oxycodone-evoked increases are dose-dependent and doses that caused increases resulted in robust and long lasting changes. (D) The low oxycodone dose $(0.1 \mathrm{mg} / \mathrm{kg}$, i.v.; yellow), caused no change in [DA]. (E) The medium oxycodone dose $(0.5 \mathrm{mg} / \mathrm{kg}$, i.v.; orange) caused a significant increase in [DA] beginning in the second time bin and lasting for the entire recording period. (F) The high oxycodone dose $(1.0 \mathrm{mg} / \mathrm{kg}$, i.v.; red) caused the same significant increase in [DA] as the medium dose, from the second time bin to the end of the recording period. (G-I) Changes in $\mathrm{pH}$ following infusions of cumulatively increasing doses of oxycodone compared to infusions of saline (doses: low to high, same colors except with white stripes). (G) The low oxycodone dose $(0.1 \mathrm{mg} / \mathrm{kg}$, i.v.) caused no change in local pH levels. ( $\mathrm{H}$ and $\mathrm{I})$ Conversely, the medium $(0.5 \mathrm{mg} / \mathrm{kg}$, i.v.) and high (1.0 mg/kg, i.v.) oxycodone doses caused a significant acidic shift in $\mathrm{pH}$ from the first time bin through the remainder of the sampling period. (J-L) The effects of oxycodone following pretreatment of either control infusions of saline or infusions of the MORpreferring antagonist naloxone $(3.0 \mathrm{mg} / \mathrm{kg}$, i.v.). (J) A representative color plot demonstrating that, following naloxone pre-treatment, oxycodone-evoked increases in DA transmission and the acidic shift in $\mathrm{pH}$ are eliminated. $(\mathrm{K})$ Quantitatively, while the medium dose of oxycodone $(0.5 \mathrm{mg} / \mathrm{kg}$, i.v. $)$ significantly increased [DA] following a saline pre-treatment, when oxycodone delivery was preceded by naloxone it no longer increased [DA]. (L) Similar to $\mathrm{DA}$, naloxone pretreatment prevented oxycodone-induced changes in acidic $\mathrm{pH}$. Error bars indicate SEM; $* P<0.05$. The i.v. infusion is indicated by a blue rectangle which is accurate according to the time and duration of saline or drug infusion. 
sion. In addition to overall changes in [DA], FSCV allows for examination of distinct neurotransmission components that contribute to overall changes in [DA] which are approximated by analysing DA transients (phasic surges in [DA]). Specifically, (i) the occurrence of phasic DA release events can be approximated by measuring transient frequency, (ii) the magnitude of DA release per phasic event is estimated from the transient amplitude, and (iii) the duration of increases of DA into the extracellular space is measured as the transient half-width. Therefore, we next analysed transient frequency, amplitude and half-width during the 15 min following each infusion to elucidate the contribution of these specific neurotransmission components for opioid-evoked increases in [DA].

\section{Transient frequency}

Similar to previous studies, control infusions of saline did not produce changes in transient frequency for morphine (Fig. 4A; $F_{14,82.910}=1.095, P=0.374$ ) or oxycodone (Fig. 4B; $F_{14,76.585}$ $=1.806, P=0.053)$. However, following morphine delivery, transient frequency was significantly increased above saline levels for the first minute after drug infusion, but not thereafter, for all doses tested (Fig. 4A; main effect of time: low dose, $F_{14,63.338}=4.774$, $P<0.001$; medium dose, $F_{14,68.048}=7.297, P<0.001$; high dose, $F_{14.58 .068}=4.895, P<0.001$; first minute after infusion: low dose, $t_{76.067}=2.762, \quad P=0.007 ;$ medium dose, $t(81.234)=6.772$, $P<0.001$; high dose, $\left.t_{71.550}=5.099, P<0.001\right)$. In contrast to morphine, minute-by-minute analysis of transient frequency follow-
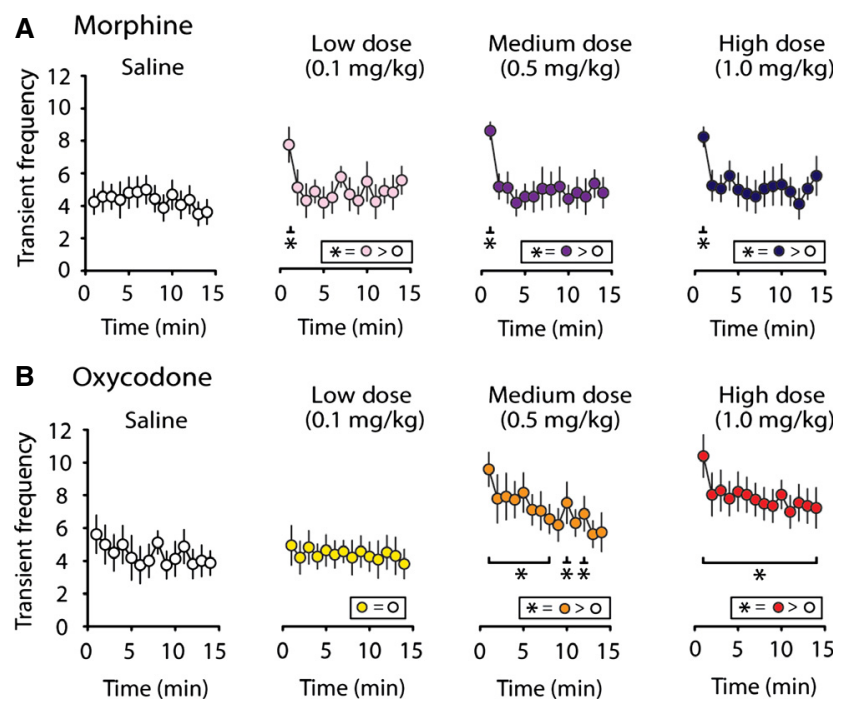

FIG. 4. Dopamine transient frequency following administration of morphine or oxycodone. (A) and (B) Transient events were binned every min for the duration of the $15 \mathrm{~min}$ recording intervals following each drug dose and compared to the average post-saline frequency. (A) Transient data per min following morphine infusions. While morphine failed to affect transient frequency across the entire 15 min recording period, all doses of morphine elicited a significant increase in transient frequency during the first minute following drug infusion compared to saline controls. (B) Oxycodone-evoked increases in DA transient frequency were highly dose-dependent. While the low dose infusion of oxycodone did not increase transient frequency, the medium dose increased DA transients for most time points and infusion of the high dose of oxycodone elevated transient frequency for the entire duration of the recording period. Error bars indicate SEM. *Indicates statistically significant increases $(P<0.05)$. ing oxycodone delivery showed a dose-dependent increase in transient frequency with the low dose causing no increase (Fig. 4B; $\left.F_{14,65.414}=0.541, P=0.899\right)$, the medium dose increasing transient frequency for most time points (Fig. 4B; $F_{14,62.633}=8.453$, $P<0.001)$ and the high dose significantly increasing transient frequency over saline controls for the entire 15 -min recording period (Fig. 4B; $F_{14,60.880}=8.103, P<0.001$ ). Thus, given that transient frequency is an approximation to the number of phasic DA release events, these data strongly suggest that oxycodone, but not morphine, causes an increase in the occurrence of phasic surges of high concentration DA release events in the NAc.

\section{Transient amplitude}

Representative color plots taken $\sim 2$ min following morphine (Fig. 5A) and oxycodone (Fig. 5B) infusions demonstrate the robust differences in transient amplitudes following delivery of these different opioids, with oxycodone evoking higher concentration transients than morphine; this is further illustrated by the insets of representative transients within these color plots (Fig. 5A and B). Again, for statistical analysis, mean amplitudes were binned across 1-min time periods (Fig. 5C and D). For both morphine (Fig. 5C; $F_{14,65.061}=0.848, \quad P=0.616$ ) and oxycodone (Fig. 5D; $\left.F_{14,65.107}=1.394, P=0.182\right)$ subjects, control saline infusions did not significantly alter mean transient amplitude compared to the baseline period. As with transient frequency, morphine caused an increase in transient amplitude within the first minute immediately following drug infusion for all doses (low dose, $t_{61.269}=2.237$, $P=0.029$; medium dose, $t_{89.211}=1.314, P<0.001$; high dose, $t_{65.760}=2.505, P=0.015$ ) but not thereafter (Fig. 5C). However, oxycodone dose-dependently increased transient amplitude: the low dose of oxycodone failed to increase transient amplitude (Fig. 5D; $\left.F_{14,58.642}=0.608, P=0.847\right)$, the medium increased amplitude for $7 \mathrm{~min}$ (Fig. 5D; main effect, $F_{14,70.005}=2.268, P=0.013$ ) and the high dose significantly increased amplitude for $11 \mathrm{~min}$ (Fig. 5D; main effect, $F_{14,75.438}=2.9905, P<0.001$ ).

\section{Transient half-width}

In contrast to transient frequency and amplitude, neither opioid robustly altered transient half-width (Fig. 6). Example [DA] traces in Fig. 6 are taken from the color plots shown in Fig. 5. Inspection of individual traces following morphine (Fig. 6A) and oxycodone (Fig. 6B) delivery, which include representative half-width values (as well as the mean for the trace), show that neither opioid substantially altered DA uptake, as determined by transient half-width values. For quantification of the data, data were binned minute-byminute in the same manner as was done for transient frequency and amplitude. This analysis shows that while saline and low dose morphine infusions did not significantly increase transient half-width, the medium dose of morphine significantly increased half-width at the 1 - and 4-min time points $\left(t_{104.945}=2.787 ; P=0.006\right)$. There was also a significant increase $1 \mathrm{~min}$ following delivery of the high dose (Fig. 6D; $t_{84.328}=2.121, P=0.037$ ). Conversely, oxycodone administration did not alter transient half-width at any time point across all doses tested (Fig. 6E; low dose, $F_{14,60.054}=0.483$, $P=0.933$; medium dose, $F_{14,64.918}=0.862, P=0.602$; high dose, $\left.F_{14,67.861}=0.673, P=0.793\right)$. Thus, in contrast to transient frequency and amplitude, transient half-width showed not robust changes following infusion of either opioid, suggesting that slowing DA uptake is not a mechanism by which opioids increase [DA] within the NAc. 

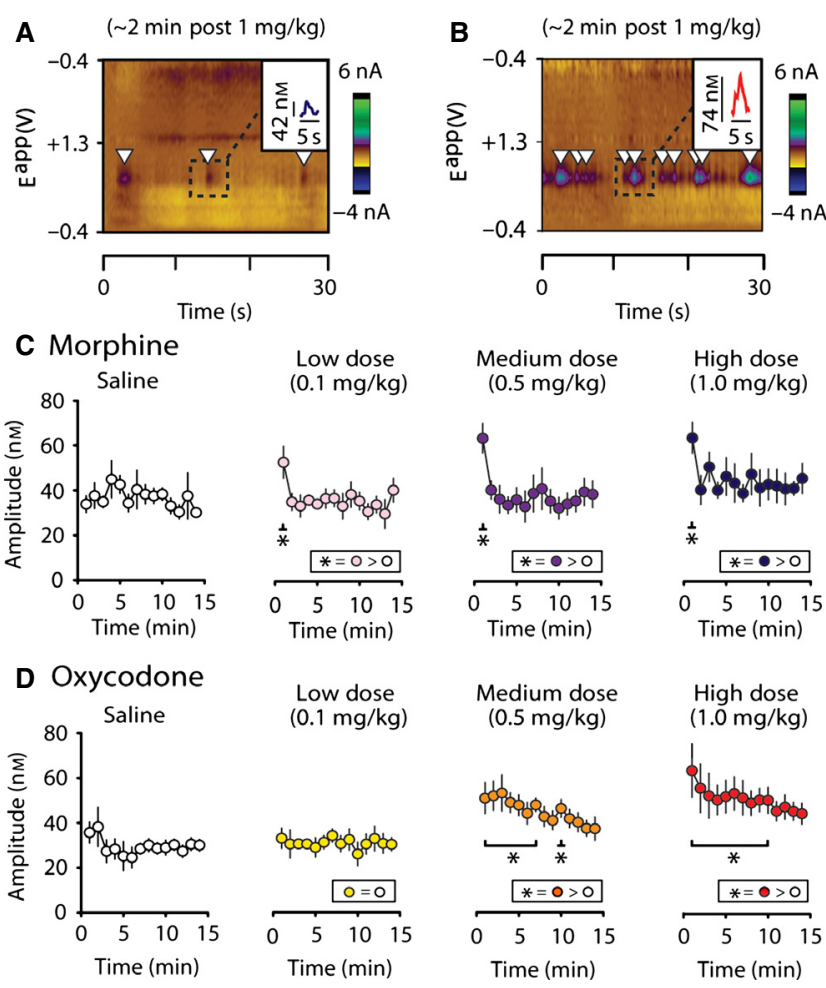

Time (min)
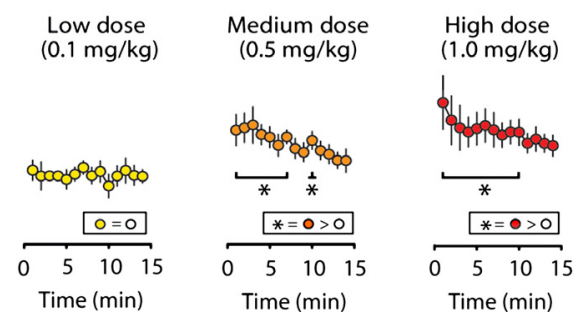

FIG. 5. Dopamine transient amplitude following administration of morphine or oxycodone. (A) A representative color plot showing a recording $\sim 2 \mathrm{~min}$ following a high dose morphine infusion within the NAc shell, and (B) a similar recording following a high dose oxycodone infusion. Insets show a zoomed in inspection of individual transients and provides their amplitudes. (C) and (D) Transient events were binned every min for the duration of the 15 min recording interval and compared to the average amplitude post-control saline infusion. (C) As with transient frequency, morphine-evoked increases in DA transient amplitude measured across 1 min time bins shows that regardless of whether subjects received low, medium or high doses of morphine, transient amplitude was significantly increased above levels of those gathered from saline controls for only the first min following morphine infusion. (D) Oxycodone-evoked increases in DA transient amplitude were dose-dependent. The low dose had no effect on transient amplitude; however, both medium and high doses of oxycodone elevated transient amplitude for the majority of the recording period. Error bars indicate SEM. *Indicates statistically significant increases $(P<0.05)$.

\section{Core-shell differences following oxycodone but not morphine delivery}

A common feature of the primary rewarding effects of drugs of abuse is that they evoke greater [DA] within the NAc shell than the NAc core (Pontieri et al., 1995; Ito et al., 2000; Frank et al., 2008). To determine whether oxycodone and morphine evoke greater $[\mathrm{DA}]$ in the shell than the core, we conducted one-half of our FSCV measurements in the NAc core and the other half in the NAc shell (Fig. 7A). While our previous studies using FSCV to examine cocaine-evoked DA transmission showed dramatic coreshell differences (Aragona et al., 2008, 2009), the present study revealed only modest core-shell differences following opioid delivery. Indeed, morphine administration, at all doses tested, produced nearly equivalent increases in $[\mathrm{DA}]$ across the NAc core and shell (Fig. 7A; main effect of subregion: low dose, $F_{1,8.427}=1.648$, $P=0.233$; medium dose, $F_{1,7.866}=0.519, P=0.492$; high dose, $\left.F_{1,6.839}<0.001, P=0.984\right)$. However, oxycodone delivery resulted in a modest, but significantly higher, increase in $[D A]$ within the NAc shell following delivery of the medium dose (from 60 to
A

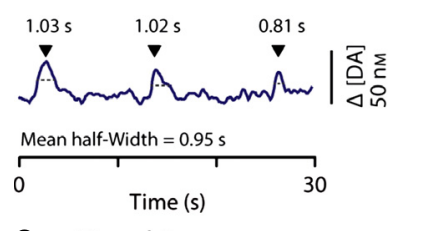

$\begin{array}{lll}\text { C } & \text { Morphine } & \text { Low dose } \\ \text { त. } & \text { Saline } & (0.1 \mathrm{mg} / \mathrm{kg})\end{array}$
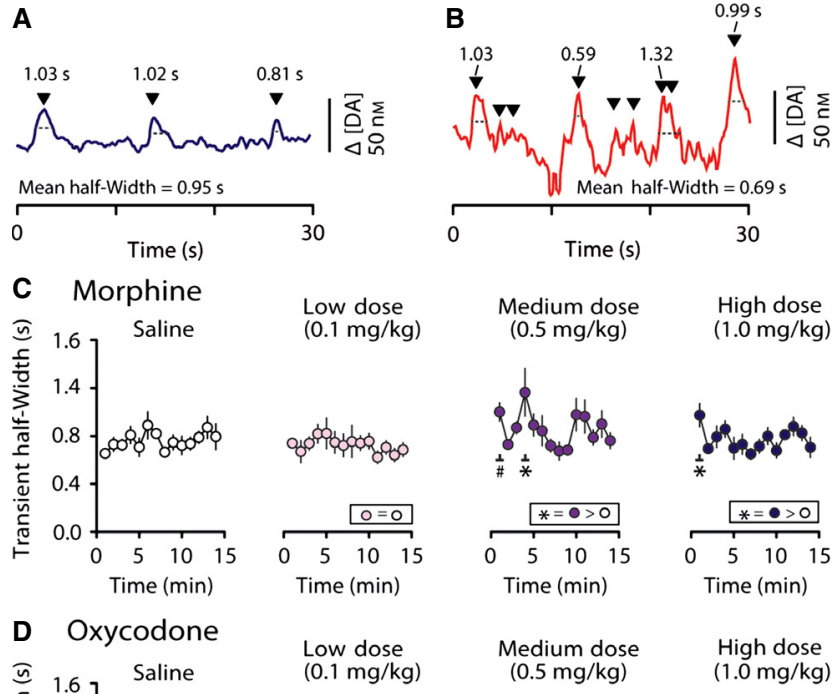

D Oxycodone

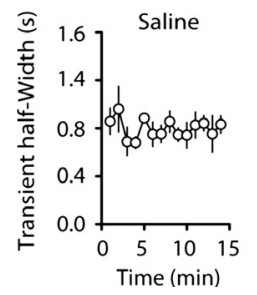

Low dose

$(0.1 \mathrm{mg} / \mathrm{kg})$

FIG. 6. Dopamine transient half-width following administration of morphine or oxycodone. (A) A representative [DA] trace (that corresponds to the color plot shown in Fig 4B) showing the recording $\sim 2$ min following high dose morphine infusion within the NAc shell, and (B) a similar [DA] trace following a high dose oxycodone infusion. (C) and (D) Mean transient half-width was binned every min for the duration of the $15 \mathrm{~min}$ recording interval and compared to the average half-width post-control saline infusion. (C) Morphine-evoked increases in DA transient half-width measured across 1 min time bins shows that only subjects that received low dose morphine infusion failed to increase transient half-width. Following medium and high dose infusions of morphine, transient half-width was significantly increased at the 3 min mark following the medium dose of morphine and during the first min following the high dose. There was a trend towards significance during the first min following the medium dose of morphine. (D) At all tested doses, oxycodone failed to increase transient half-width. Error bars indicate SEM. *indicates statistically significant increases $(P<0.05)$. " Indicates trending increase $(P<0.09)$.

$120 \mathrm{~s} ;$ Fig. $7 \mathrm{~B} ; t_{71.577}=-2.302, P=0.024$ for bin 6 , and continuing $P<0.001$ for bins 7-12) and the high dose of oxycodone (during the last two time bins, $100-120 \mathrm{~s}, t_{65.738}=2.060$, $P=0.043$, and $t_{63.350}=2.197, P=0.032$ ). In addition to causing a greater increase in $[\mathrm{DA}]$ in the NAc shell, the medium and high (but not the low) doses of oxycodone caused greater increases in the frequency and amplitude of DA transients within the NAc shell than in the core (Table 1).

A positive control to test for electrode fidelity was the infusion of the DA autoreceptor blocker raclopride at a dose $(1.0 \mathrm{mg} / \mathrm{kg})$ known to induce burst firing and forebrain DA release (Andersson et al., 1995). Consistent with previous studies, [DA] levels were significantly higher within the NAc shell than the NAc core across multiple time points (Fig. 8C and F; $t_{116.807}=-2.043, P=0.043$; $\left.t_{107.583}=-2.112, P=0.037 ; t_{99.064}=-2.601, P=0.011\right)$. Thus, these data demonstrate that drugs that cause DA neuron firing, presumably equally into the NAc core and shell, result in a more modest preferential enhancement of [DA] within the NAc shell which is probably due to reduced uptake in the NAc shell compared to the core (Aragona et al., 2008). 
A
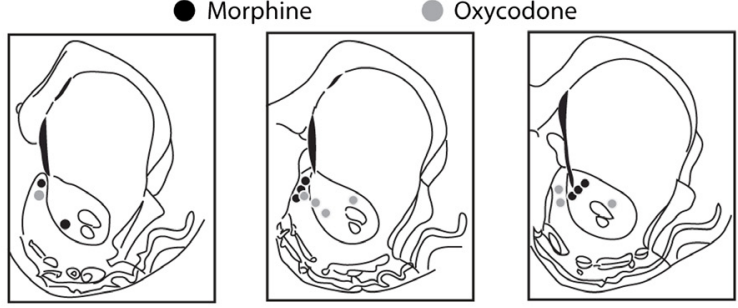

B Morphine

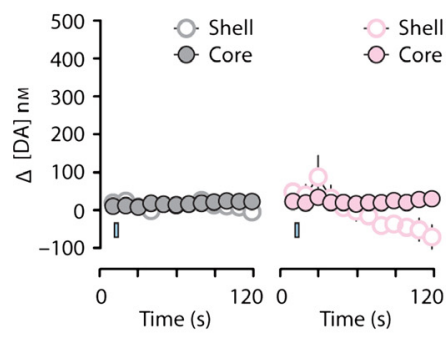

Shell

O Shell

Core

O Shell

Core

C Oxycodone

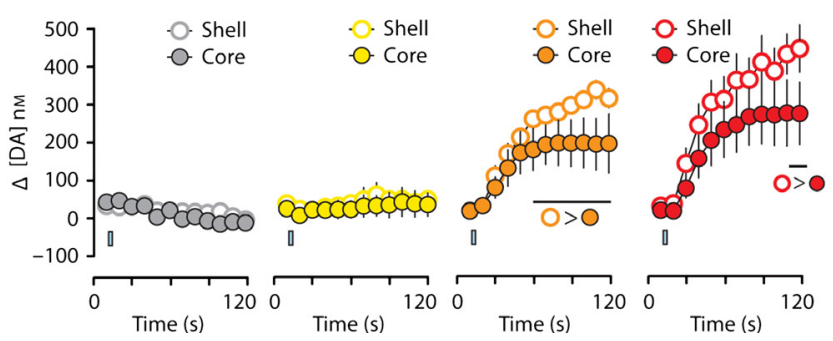

FIG. 7. Morphine and oxycodone, in general, do not evoke greater dopamine release in the NAc shell compare to the core. (A) Histology for both morphine subjects $(n=8)$ recorded from the NAc core $(n=4)$ or shell $(n=4)$ and oxycodone subjects $(n=8)$ - NAc core $(n=4)$ or shell $(n=4)$ - were recorded from similar locations within the NAc. (B) [DA] for morphine subjects were binned every $10 \mathrm{~s}$ across the $120 \mathrm{~s}$ sampling window encompassing i.v. infusions (from left to right: saline; $0.1 \mathrm{mg} / \mathrm{kg} ; 0.5 \mathrm{mg} / \mathrm{kg}$; $1.0 \mathrm{mg} / \mathrm{kg}$ ). Morphine did not significantly elevate [DA] in the NAc shell [open circles] compared to the core [closed circles]. (C) [DA] for oxycodone subjects were binned every $10 \mathrm{~s}$ across the $120 \mathrm{~s}$ sampling window encompassing i.v. infusions (from left to right: saline; $0.1 \mathrm{mg} / \mathrm{kg} ; 0.5 \mathrm{mg} / \mathrm{kg}$; $1.0 \mathrm{mg} / \mathrm{kg}$ ). Oxycodone did not significantly elevate [DA] in the NAc shell [open circles] compared to the core [closed circles] at the low $0.1 \mathrm{mg} / \mathrm{kg}$ dose. Conversely, oxycodone caused a modest, but significant increase in [DA] within the NAc shell compared to the core at the medium $(0.5 \mathrm{mg} / \mathrm{kg})$ and high $(1.0 \mathrm{mg} / \mathrm{kg})$ doses. Error bars indicate SEM. The i.v. infusion is indicated by a blue rectangle which is accurate according to the time and duration of saline or drug infusion.

\section{Rapid microdialysis with mass spectrometry revealed coincident peaks in dopamine and GABA concentration following morphine, but not oxycodone, delivery, consistent with the FSCV results}

The sub-second measurements provided by FSCV described above revealed that morphine and oxycodone evoked very different phasic

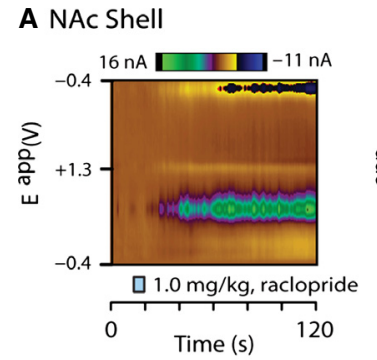

B NAc Core
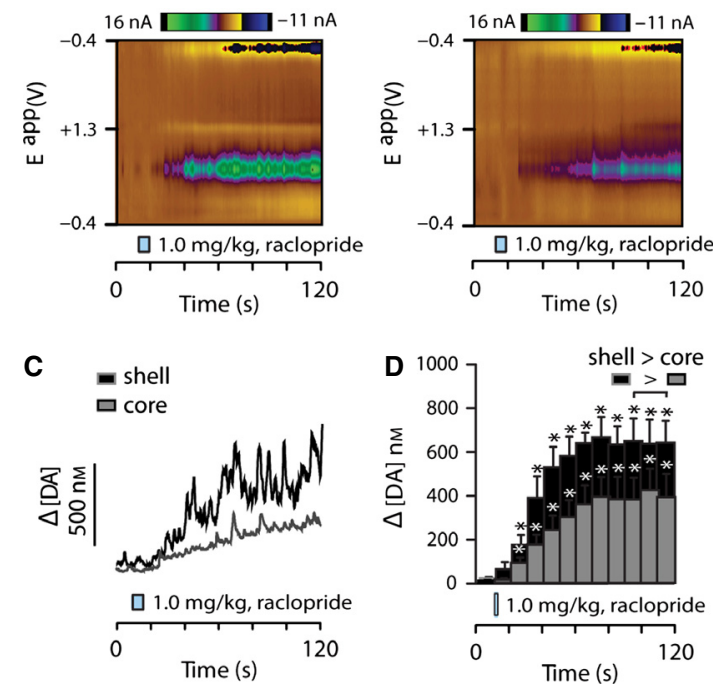

FIG. 8. Raclopride evokes greater dopamine concentration within the NAc shell compared to the NAc core. (A) A representative color plot of increased DA transmission within the NAc shell following i.v. raclopride infusion $(1.0 \mathrm{mg} / \mathrm{kg}$; which followed the opioid infusions described above). (B) A representative color plot is shown from the NAc core following the same dose of raclopride. (C) Corresponding [DA] traces associated with the color plots. [DA] is robustly elevated raclopride infusion in both the core and shell. The resulting [DA] traces associated with these color plots show that autoreceptor blockade causes an increase in DA transients superimposed on a gradual rise in [DA]. (D) Quantitative data of raclopride-evoked increases in [DA] within the NAc core and shell. Despite the similarities in the nature of raclopride-evoked increases in phasic DA release, increased $[\mathrm{DA}]$ in the shell is significantly higher compared to that evoked within the NAc core from 30 to $120 \mathrm{~s}$ following raclopride infusion. Error bars indicate SEM. *Indicates statistically significant increases $(P<0.05)$.

patterns in DA transmission in the NAc. However, FSCV is optimised for measuring phasic changes in $\mathrm{DA}$ and $\mathrm{pH}$. Therefore we employed rapid microdialysis coupled with HPCL-MS (1-min sampling resolution) to measure 16 compounds following morphine and oxycodone delivery (Fig. 9A and B). Another limitation of FSCV is that the background subtraction results in the inability to measure changes in [DA] over long periods of time (i.e., over many minutes). Again, microdialysis with HPLC-MS allows minute-by-minute changes after drug delivery for many chemical species (Fig. 9A and B) (Song et al., 2012).

With respect to DA transmission within the NAc (Fig. 9C) following opioid delivery, the microdialysis HPLC-MS data are consistent with the FSCV data shown above. While saline infusions (i.v.) did not significantly alter [DA] (Fig. 9D), i.v. infusion of oxycodone produced a significant increase in [DA], compared to baseline and saline infusion levels, that lasted the duration of the recording period (Fig. 9D; main effect, $F_{40,69.766}=1.693, P=0.027$ ). In contrast, the significant morphine increase was brief; [DA] was elevated only

TABLE 1. Frequency and amplitude of DA transients in the NAc shell vs. the core following i.v. oxycodone

\begin{tabular}{|c|c|c|c|c|c|c|c|c|}
\hline & \multicolumn{4}{|c|}{ Transient frequency } & \multicolumn{4}{|c|}{ Transient amplitude } \\
\hline & Shell & Core & $F_{1,18}$ & $P$ & Shell & Core & $F_{1,16}$ & $P$ \\
\hline Medium & $9.5 \pm 0.3$ & $5.0 \pm 1.1$ & 11.234 & 0.004 & $54.3 \pm 3.4$ & $35.9 \pm 2.0$ & 12.010 & 0.003 \\
\hline High & $10.2 \pm 0.5$ & $5.8 \pm 4.0$ & 10.580 & 0.004 & $65.3 \pm 5.4$ & $38.0 \pm 4.0$ & 22.649 & $<0.001$ \\
\hline
\end{tabular}


A

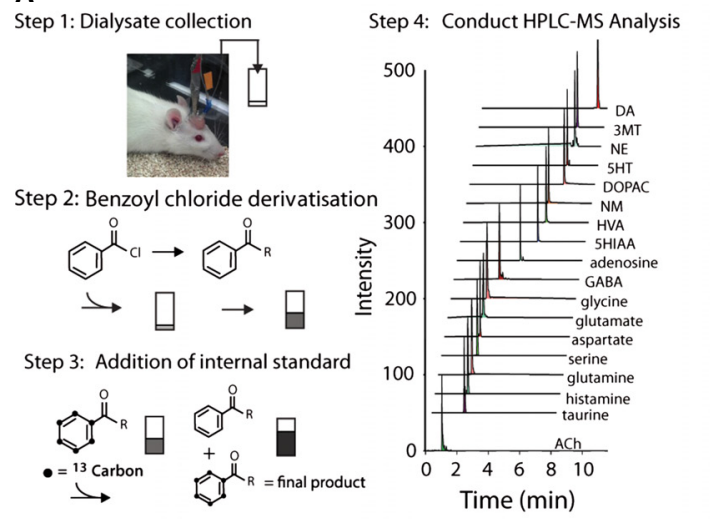

c
B
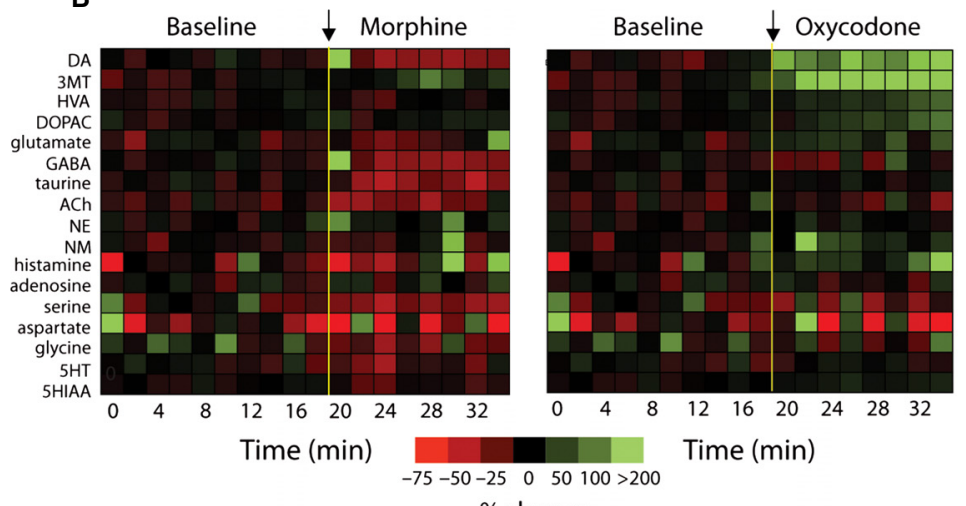

$-75-50-25 \quad 0 \quad 50100>200$

$\%$ change
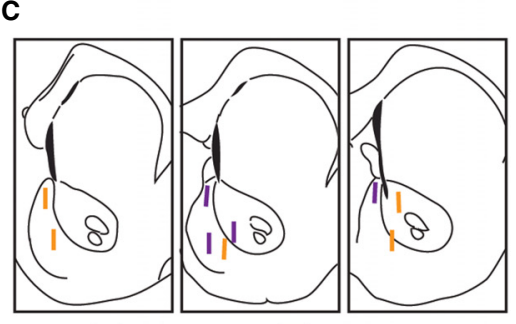

Oxycodone
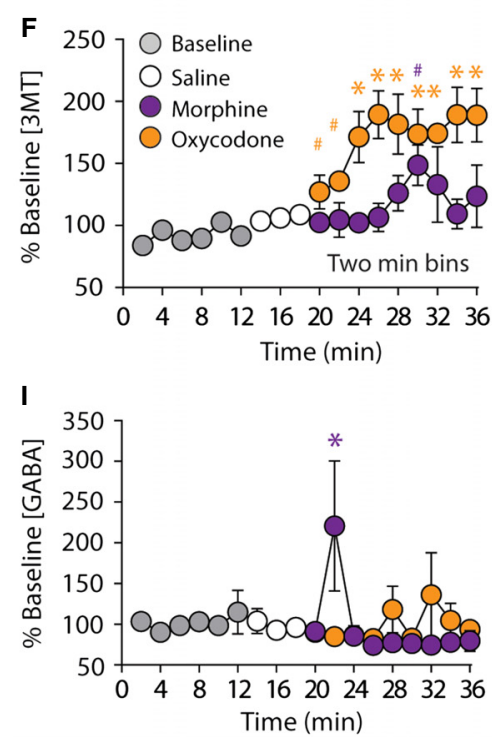

D 250
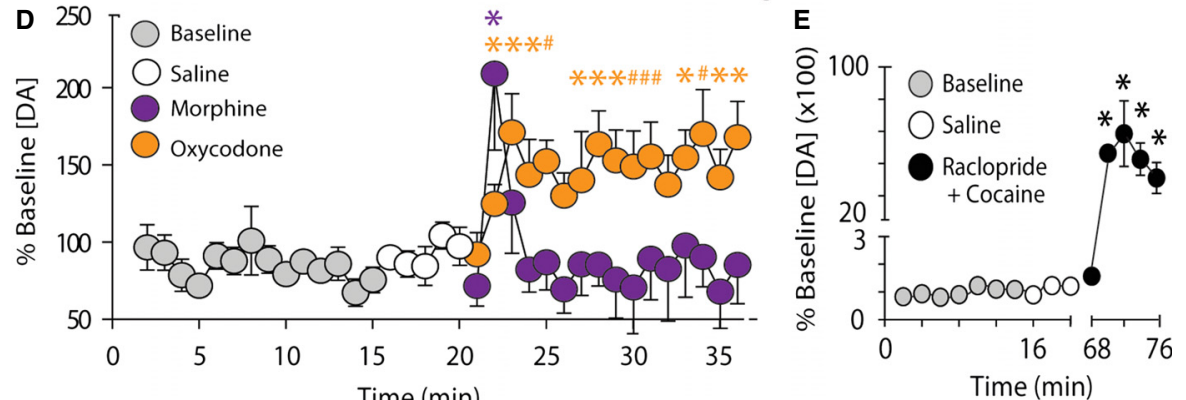
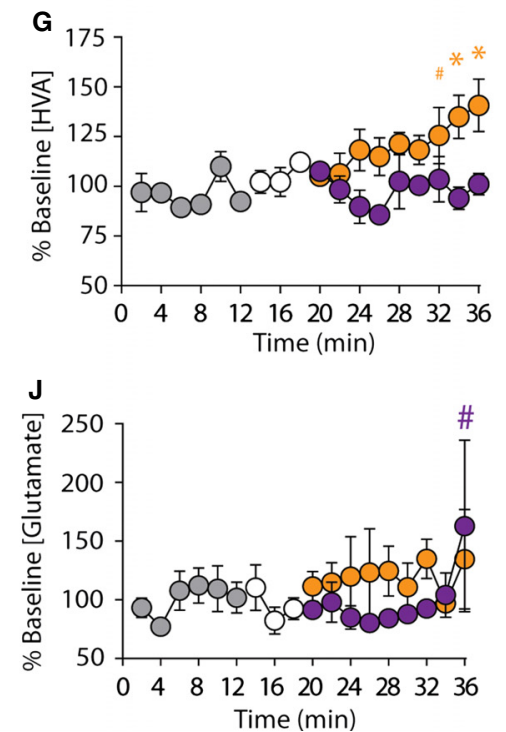
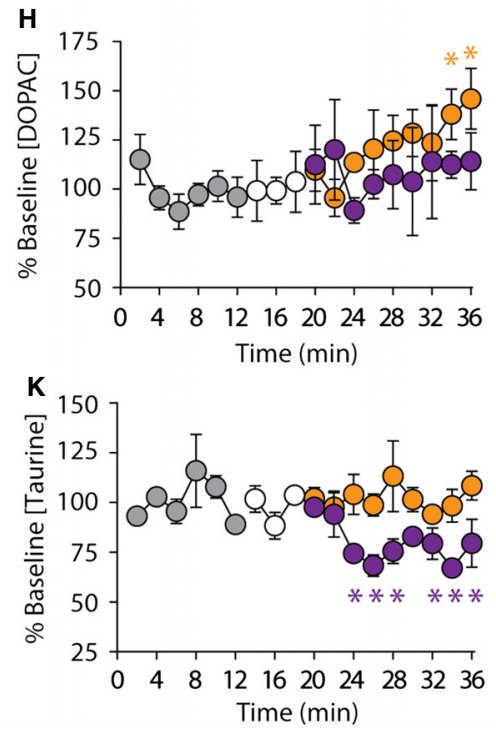

FIG. 9. Rapid sampling microdialysis coupled with HPLC-MS reveals differential changes in dopamine transmission within the NAc between morphine and oxycodone. (A) Dialysate was collected every minute (step 1) and immediately derivatised with benzoyl chloride (step 2). Internal standards were added to improve quantification (step 3), and fractions were analysed with HPLC-MS (step 4). A representative total ion chromatogram of a dialysate sample reveals that a tremendous numbers of analytes can be measured using this technology compared to traditional microdialysis. (B) Heatmap representing mean percentage changes under baseline conditions and changes following i.v. morphine $(0.5 \mathrm{mg} / \mathrm{kg} ; n=4)$ and oxycodone $(0.5 \mathrm{mg} / \mathrm{kg}$, i.v.; $n=5)$ delivery. (C). Histological representation of all probe placements for subjects receiving morphine (purple) and oxycodone (orange). (D) Normalised changes in [DA] within the NAc (core and shell combined). Baseline levels are shown in gray and control i.v. infusions of saline (white circles) did not significantly increase [DA] above basal levels. Infusion of morphine $(0.5 \mathrm{mg} / \mathrm{kg}$ i.v.; purple circles) significantly increased [DA] for $1 \mathrm{~min}$; levels then returned to baseline. Consistent with FSCV data, oxycodone (orange circles) caused a significant increase in [DA] immediately and remained elevated for the entire sampling period. (E) As a positive control, a drug cocktail known to robustly increase [DA] (the D2 antagonist raclopride and the DAT blocker cocaine) evoked an extremely robust increase in [DA] ( 5000\%). (F) Consistent with changes in [DA], the DA metabolite 3-MT trended toward significance in the first two recording blocks following oxycodone administration, and was significantly increased during the remainder of the recording session. However, following morphine, 3-MT was largely unchanged following morphine delivery with the exception of a trend toward significance in the sixth recording session. (G) Following oxycodone, the DA metabolite HVA showed a trend toward significance in the seventh session and was significantly increased in the final two recording bins. However, HVA levels remained unchanged following morphine delivery. (H) The DA metabolite DOPAC was significantly elevated in the final two recording bins following oxycodone delivery, but showed no change following morphine infusion. (I) The only neurotransmitter that showed a similar pattern of transmission that was similar to DA was GABA transmission following morphine delivery. GABA was significantly elevated following morphine, but not oxycodone, infusion and this increase was only significant during the first time point following drug infusion. ( $\mathrm{J}$ ) There was a trend toward a significant increase in glutamate concentration in the final recording session following morphine but not oxycodone delivery. (K) Morphine infusion significantly decreased taurine concentration, beginning at the third time bin, and remained decreased for the duration of the recording period. Conversely, oxycodone infusion had no effect on taurine levels. Error bars indicate SEM; ${ }^{*} P<0.05 ;{ }^{*} P<0.09$. 
during the first minute immediately following drug administration $\left(t_{107.996}=5.303, P<0.001\right)$. Finally, we used a positive control to demonstrate the validity of these rapid dialysis measures. A combination of the D2 DA receptor antagonist raclopride and the DA transporter blocker, cocaine, which has been shown to cause a synergistic increase in [DA] (Rouge-Pont et al., 2002; Aragona et al., 2008), was administered. Consistent with these previous dialysis studies, cocaine in the presence of raclopride caused an extremely large increase in DA release (Fig. 9E; $P<0.05$, minutes 70-76).

Changes in concentrations of DA metabolites are consistent with the morphine-oxycodone differences observed for [DA] (Fig. 9F-H). Specifically, oxycodone, but not morphine, caused increases in the DA metabolites 3-MT (Fig. 9F; oxycodone, $F_{9,14.670}=2.596$, $P=0.051$; morphine, $F_{9,5.623}=0.879, P=0.589$ ), HVA (Fig. 9G; last two oxycodone bins respectively, $t_{23.020}=2.645, P=0.014$ and $t(22.939)=3.079, \quad P=0.005 ; \quad$ morphine, $\quad F_{9,11.182}=1.030$, $P=0.473$ ), and DOPAC (Fig. 9H; last two oxycodone bins respectively, $t_{29.887}=2.080, P=0.046$ and $t_{29.887}=2.510, P=0.018$; morphine, $\left.F_{9,12.910}=0.325, P=0.951\right)$. Microdialysis with HPLCMS provides the opportunity to investigate simultaneous changes in multiple neurotransmitter concentrations following morphine and oxycodone. As described above, morphine, but not oxycodone, caused a brief increase in [DA] that rapidly returned to baseline and this result was observed using both FSCV and microdialysis (see above). It is therefore interesting that morphine, but not oxycodone, delivery produced a coincident increase in GABA concentration during the same time point of the initial peak in [DA] following drug delivery but not thereafter (Fig. 9I; $t_{29.99}=3.218, \quad P=0.003$ ). Given GABA's inhibitory role in neurotransmission, this increase in GABA concentration following morphine may be responsible for DA returning to baseline immediately after its initial peak following delivery. This brief morphine-evoked increase in GABA within the NAc is probably involved in explaining how these two opioids differentially mediate reward.

In contrast to GABA, there was no significant change in glutamate concentration following delivery of either drug except for a trend for an increase toward the end of the sampling period following morphine delivery (Fig. 9J). Additionally, there was a significant and sustained decrease in taurine concentration following morphine $\left(F_{9,9.855}=3.412, P=0.035\right)$ but not oxycodone $\left(F_{9,18.410}=0.521\right.$, $P=0.840$ ) delivery (Fig. 9K). This decrease in taurine is interesting because it is consistent with previous studies showing that alcohol administration also alters NAc taurine levels (Dahchour et al., 1994).

Regarding the remainder of the analytes detected, there were no statistically significant differences in neurotransmission between morphine and oxycodone delivery (data not shown), and neither drug caused statistically significant differences in detected dialysate levels of acetylcholine (oxycodone, $F_{9,27.757}=1.977, P=0.081$; morphine, $\left.F_{9,10.732}=0.953, \quad P=0.522\right), \quad$ serotonin (oxycodone, $\quad F_{9,6.311}=$ $0.381, P=0.908$; morphine, $F_{9,4.945}=1.170, P=0.455$ ), the serotonin metabolite 5-hydroxyindole-3-acetic acid (oxycodone, $F_{9,6.173}=$ $0.553, P=0.797$; morphine, $F_{9,11.631}=1.043, P=0.463$ ), norepinephrine (oxycodone, $F_{9,17.258}=0.658, \quad P=0.735$; morphine, $\left.F_{9,11.545}=0.429, P=0.894\right)$, the norepinephrine metabolite normetanephrine (oxycodone, $F_{9,17.326}=0.964, \quad P=0.500 ; \quad$ morphine, $F_{9,6.143}=0.805, P=0.630$ ), serine (oxycodone, $F_{9,17.352}=1.012$, $P=0.467$; morphine, $F_{9,13.201}=0.711, P=0.691$ ), adenosine (oxycodone, $F_{9,16.407}=0.297, P=0.965 ;$ morphine, $F_{9,11.320}=1.253$, $P=0.354$ ), histamine (oxycodone, $F_{9,5.829}=1.509, \quad P=0.321$; morphine, $F_{9,4.971}=0.838, P=0.616$ ) or aspartate (oxycodone,
$F_{9,18.268}=1.217, \quad P=0.343 ; \quad$ morphine $, \quad F_{9,12.052}=0.943, \quad P=$ 0.525). Data are represented in heat maps in Fig. 9B.

\section{Discussion}

Prescription opioid abuse is a rapidly growing epidemic (Compton \& Volkow, 2006; McCabe et al., 2013b). In particular, oxycodone is among the most commonly abused opioids (McCabe et al., 2013a,b). While there is no doubt that greater availability of this prescription drug has contributed to this rise in abuse, there is growing evidence suggesting that oxycodone may be more potent with respect to its ability to promote addictive behaviors (Stoops et al., 2010; Comer et al., 2013). For example, in the clinical realm, oxycodone administration to human heroin addicts, compared to other opioids, caused robust reinforcing effects with no increases in negative effects (Comer et al., 2008). Further, in the same study, a verbal report from a heroin-dependent individual stated that oxycodone is the 'Rolls Royce' of opioids and that it produces a 'smooth high' (Comer et al., 2008). While recent studies have begun to investigate the influence of oxycodone on behavior and brain processes (Zhang et al., 2009, 2014; Niikura et al., 2013; Mayer-Blackwell et al., 2014), it has remained unclear whether this drug differentially alters neurotransmission associated with reward and motivation compared to other opioids. Here, we demonstrate that DA transmission within the NAc, a key neurobiological component of motivation, is starkly different following oxycodone compared to morphine delivery. Moreover, we reveal that morphine delivery is associated with a coincident surge in DA and GABA concentration immediately following drug delivery in the NAc. This increase in GABA concentration observed following morphine, but not oxycodone, may explain why [DA] quickly returns to baseline levels following delivery of morphine but not of oxycodone.

Using two rapid neurochemical measurement technologies, we reveal robust oxycodone-evoked increases in [DA]. The present FSCV experiment detected that [DA]s achieve levels as high as $500 \mathrm{~nm}$ in the NAc shell, and the microdialysis study shows that oxycodone-evoked increase in [DA] is stable and long-lasting. Indeed, drug-evoked increases in [DA] as well as DA transients remain elevated throughout the 15 -min recording period. Conversely, both the microdialysis and FSCV experiments reveal that morphine evokes an initial rapid increase in [DA] only during the first minute following drug infusion. Importantly, this morphineevoked increase in [DA] was coincident with an increase in GABA concentration, which was also elevated only within the first 1-min time bin following drug infusion.

Greater increases in [DA] following oxycodone than following morphine delivery was also associated with a greater frequency and amplitude of phasic DA release events (DA transients) but was not associated with slowed reuptake as measured by transient half-width. DA transients originate from burst firing of DA-containing neurons within the midbrain (Aragona et al., 2008; Sombers et al., 2009; Owesson-White et al., 2012). This finding that oxycodone caused a dramatic increase in DA transients is of interest because strong arguments continue to be made that the ability of a drug to potentiate DA transients is closely associated with a drug's potential risk for addiction (Covey et al., 2014).

Despite both being MOR agonists, morphine and oxycodone have distinct pharmacokinetic and pharmacodynamic properties that may contribute to their different effects on DA transmission (Nielsen et al., 2007; Lemberg et al., 2009). Although oxycodone has a lower affinity for the MOR than morphine (Chen et al., 1991; Lalovic et al., 2006), oxycodone crosses the blood-brain barrier more 
readily than morphine (Villesen et al., 2006), and unbound concentrations of oxycodone are higher in the brain than in the blood following i.v. administration, suggesting that oxycodone is actively transported across the blood-brain barrier (Boström et al., 2006, 2008; Villesen et al., 2006). Indeed, for the same unbound concentrations of oxycodone and morphine in the blood, unbound oxycodone in the brain is as much as six times higher than morphine (Boström et al., 2008). Additionally, ligand-directed signaling could contribute to differences in drug-evoked DA transmission, as morphine and oxycodone have different intrinsic efficacies and receptor internalisation profiles (Lester \& Traynor, 2006; Peckham \& Traynor, 2006; Melief et al., 2010). Finally, morphine and oxycodone have different metabolic pathways, and the actions of their respective metabolites differ, which may potentially contribute to differential drug-evoked [DA]s (Kalso, 2007). Future studies are needed to investigate the roles of these different pharmacokinetic and pharmacodynamic properties of morphine and oxycodone on altering DA transmission.

Mechanistically, as the coincident increase in GABA concentration was associated with morphine, but not oxycodone, delivery, it is an intriguing possibility that the surge in GABA could be directly related to the rapid return of DA to its baseline. In other words, GABA may mediate the inhibitory mechanism which rapidly shuts down the initial morphine-evoked increases DA transmission. Thus, the present neurochemical data provide a potential mechanism regarding differences in opioid-evoked changes in [DA] between morphine and oxycodone. There are at least two possible mechanisms whereby GABA inhibits DA transmission in the NAc. First, as local perfusion of morphine into the NAc increases GABAergic signaling (Sun et al., 2011), it is possible that increased GABA levels in the NAc may activate $\mathrm{GABA}_{\mathrm{A}}$ receptors on DA terminals and thereby reduce DA release (Aono et al., 2008; Saigusa et al., 2008). In contrast, oxycodone may promote greater MOR activation of GABAergic interneurons, reducing local GABA levels and subsequently disinhibiting DA release from terminals (Aono et al., 2008).

Second, the fact that morphine, but not oxycodone, increases GABA transmission within the NAc may be due to increased morphine-induced firing of GABAergic neurons in the VTA projecting to the NAc (van Zessen et al., 2012). This is an intriguing possibility because previous studies have shown that a single injection of morphine can produce a conditioned place aversion in drug-naïve rats (Parker et al., 2002; but see Fenu et al., 2006). There are numerous anecdotal reports describing how opioids are initially aversive before they become rewarding, and this initial aversion may involve a GABAergic mechanism. Recent studies have carefully identified that place aversions are indeed mediated by activation of these GABAergic projection neurons from the VTA to the NAc (Tan et al., 2012). Regardless of the specific mechanism, the present neurochemical data provide novel explanatory power regarding why morphine has long been known to be initially aversive and may further explain why oxycodone is a preferred opioid (Comer et al., 2008).

Previous work has suggested that activation of MORs on GABAergic interneurons within the VTA deactivate these interneurons, resulting in the disinhibition of DA neuron firing and an increase in DA neuron burst firing, and elevated [DA] release from forebrain terminals (Gysling \& Wang, 1983; Johnson and North, 1992, Matsui and Williams, 2011). However, there are additional mechanisms that remain to be elucidated as oxycodone, but not morphine, causes a substantial increase in DA transients, and previous studies have shown that morphine delivery causes an overall increase DA neuron firing without increasing burst firing (Gysling \& Wang, 1983). Furthermore, local application of morphine into the VTA increases DA neuron firing rate but this is subsequently followed by a decrease in firing rate (Matthews \& German, 1984). These electrophysiology findings are consistent with the present data, except that the aforementioned electrophysiology study did not report an initial morphine-induced burst of DA neuron activity that would coincide with the rapid morphineevoked DA release observed in the first minute post-infusion. A likely explanation for this disconnect is that most of the electrophysiology experiments conducted decades ago only recorded from 'traditional' DA neurons. Now, however, functionally and anatomically distinct sub-populations of DA neurons with different firing properties have been discovered (Ikemoto, 2007; Lammel et al., 2008, 2011). Interestingly, a subset of DA neurons along the midline of the VTA are far more susceptible to bursting (Lammel et al., 2008) and therefore are a strong candidate to be the DA neurons responsible for this initial morphine-evoked DA release. Future studies are needed to test this hypothesis.

As discussed above, it is possible that morphine-evoked increases in GABA concentration mediate early aversive processing upon initial morphine exposure. However, morphine can also be highly rewarding. Indeed, activation of MORs can promote consumption of palatable foods and has been linked to pleasure (Will et al., 2003; Kelley, 2004; Berridge \& Kringelbach, 2013). Therefore, future studies are needed to determine how increases in GABA concentrations in the NAc differentially contribute to morphine reward compared to oxycodone reward.

\section{Conclusion}

This is the first study to compare the initial rapid neurochemical changes in the NAc core and shell following delivery of two highly abused opioids, morphine and oxycodone. FSCV and rapid microdialysis HPLC-MS revealed that oxycodone caused a greater increase in [DA] in the NAc than did morphine. Oxycodone robustly increased $[D A]$ in both sub-regions of the NAc, albeit greater within the NAc shell. These increases lasted the entire recording period and, in addition to increasing [DA], oxycodone robustly increased the frequency and amplitude of DA transients. However, with the exception of increasing DA metabolites, rapid microdialysis showed that oxycodone caused no appreciable change to other signaling molecules. Conversely, morphine caused a coincident surge in DA and GABA concentration within the first minute following drug delivery but not thereafter. However, despite these differences both opioids are highly addictive when abused. As stated above, there is growing evidence that oxycodone appears to be a preferred opioid of abuse and its abuse continues to increase tremendously. Thus, it is essential to understand how drugs differentially alter neurochemistry if we hope to provide relief to addicts, and this begins by using in vivo tools, such as those described herein, to reveal how different drugs differentially impact brain signaling systems that powerfully takeover behavior.

\section{Acknowledgements}

The authors would like to thank Brenann E. Couturier, Myranda Bryan, Arif Hamid and Bianca Jenkins for technical assistance, Shanna L. Resendez for helpful discussion and Richard B. Keithley for analysis consultation and the MATLAB transient detection program. This work was supported by NIDA PO1DA031656 (B.J.A.), R37 EB00330 (R.T.K.) and the Martha Muenzer Memorial Award (C.M.V.W.), and K.A.P.S. was supported by NIH T32 DA007267. The authors declare no competing financial interests. 


\section{Abbreviations}

3-MT, 3-methoxytyramine; CV, cyclic voltammogram; DA, dopamine; [DA], extracellular DA concentration; DOPAC, 3,4-dihydroxyphenylacetic acid; FSCV, fast-scan cyclic voltammetry; HPLC-MS, high-performance liquid chromatography coupled with mass spectrometry; HVA, homovanillic acid; MOR, $\mu$-opioid receptor; NAc, nucleus accumbens; VTA, ventral tegmental area.

\section{References}

Andersson, J.L., Nomikos, G.G., Marcus, M., Hertel, P., Mathe, J.M. \& Svensson, T.H. (1995) Ritanserin potentiates the stimulatory effects of raclopride on neuronal activity and dopamine release selectivity in the mesolimbic dopaminergic system. N.-S. Arch. Pharmacol., 352, 374385.

Aono, Y., Saigusa, T., Mizoguchi, N., Iwakami, T., Takada, K., Gionhaku, N., Oi, Y., Ueda, K., Koshikawa, N. \& Cools, A.R. (2008) Role of $\mathrm{GABA}_{\mathrm{A}}$ receptors in the endomorphin-1-, but not endomorphin-2-, induced dopamine efflux in the nucleus accumbens of freely moving rats. Eur. J. Pharmacol., 580, 87-94.

Aragona, B.J., Cleaveland, N.A., Stuber, G.D., Day, J.J., Carelli, R.M. \& Wightman, R.M. (2008) Preferential enhancement of dopamine transmission within the nucleus accumbens shell by cocaine is attributable to a direct increase in phasic dopamine release events. J. Neurosci., 28, 88218831

Aragona, B.J., Day, J.J., Roitman, M.F., Cleaveland, N.A., Wightman, R.M. \& Carelli, R.M. (2009) Regional specificity in the real-time development of phasic dopamine transmission patterns during acquisition of a cuecocaine association in rats. Eur. J. Neurosci., 30, 1889-1899.

Badrinarayan, A., Wescott, S.A., Vander Weele, C.M., Saunders, B.T., Couturier, B.E., Maren, S. \& Aragona, B.J. (2012) Aversive stimuli differentially modulate real-time dopamine transmission dynamics within the nucleus accumbens core and shell. J. Neurosci., 32, 15779-15790.

Berridge, K.C. \& Kringelbach, M.L. (2013) Neuroscience of affect: brain mechanisms of pleasure and displeasure. Curr. Opin. Neurobiol., 23, 294303.

Boström, E., Simonsson, U.S. \& Hammarlund-Udenaes, M. (2006) In vivo blood-brain barrier transport of oxycodone in the rat: indications for active influx and implications for pharmacokinetics/pharmacodynamics. Drug Metab. Dispos., 34, 1624-1631.

Boström, E., Hammarlund-Udenaes, M. \& Simonsson, U.S. (2008) Bloodbrain barrier transport helps to explain discrepancies in in vivo potency between oxycodone and morphine. Anesthesiology, 108, 495-505.

Cheer, J.F., Wassum, K.M., Sombers, L.A., Heien, M.L., Ariansen, J.L., Aragona, B.J., Phillips, P.E. \& Wightman, R.M. (2007) Phasic dopamine release evoked by abused substances requires cannabinoid receptor activation. J. Neurosci., 27, 791-795.

Chen, Z.R., Irvine, R.J., Somogyi, A.A. \& Bochner, F. (1991) Mu receptor binding of some commonly used opioids and their metabolites. Life Sci., 48, 2165-2171

Comer, S.D., Sullivan, M.A., Whittington, R.A., Vosburg, S.K. \& Kowalczyk, W.J. (2008) Abuse liability of prescription opioids compared to heroin in morphine-maintained heroin abusers. Neuropsychopharmacology, 33 1179-1191.

Comer, S.D., Metz, V.E., Cooper, Z.D., Kowalczyk, W.J., Jones, J.D., Sullivan, M.A., Manubay, J.M., Vosburg, S.K., Smith, M.E., Peyser, D. \& Saccone, P.A. (2013) Comparison of a drug versus money and drug versus drug self-administration choice procedure with oxycodone and morphine in opioid addicts. Behav. Pharmacol., 24, 504-516.

Compton, W.M. \& Volkow, N.D. (2006) Major increases in opioid analgesic abuse in the United States: concerns and strategies. Drug Alcohol Depen., 81, 103-107.

Covey, D.P., Roitman, M.F. \& Garris, P.A. (2014) Illicit dopamine transients: reconciling actions of abused drugs. Trends Neurosci., 37, 200-210.

Daberkow, D.P., Brown, H.D., Bunner, K.D., Kraniotis, S.A., Doellman, M.A., Ragozzino, M.E., Garris, P.A. \& Roitman, M.F. (2013) Amphetamine paradoxically augments exocytotic dopamine release and phasic dopamine signals. J. Neurosci., 33, 452-463.

Dahchour, A., Quertemont, E. \& De Witte, P. (1994) Acute ethanol increases taurine but neither glutamate nor GABA in the nucleus accumbens of male rats: a microdialysis study. Alcohol Alcoholism, 29, 485-487.

Davis, M.P., Varga, J., Dickerson, D., Walsh, D., LeGrand, S.B. \& Lagman, R. (2003) Normal-release and controlled-release oxycodone: phar- macokinetics, pharmacodynamics, and controversy. Support. Care Cancer, 11, 84-92.

Di Chiara, G. \& Imperato, A. (1988) Drugs abused by humans preferentially increase synaptic dopamine concentrations in the mesolimbic system of freely moving rats. Proc. Natl. Acad. Sci. USA, 85, 5274-5278.

Fenu, S., Spina, L., Rivas, E., Longoni, R. \& Di Chiara, G. (2006) Morphine-conditioned single-trial place preference: role of nucleus accumbens shell dopamine receptors in acquisition, but not expression. Psychopharmacology, 187, 143-153.

Frank, S.T., Krumm, B. \& Spanagel, R. (2008) Cocaine-induced dopamine overflow within the nucleus accumbens measured by in vivo microdialysis: a meta-analysis. Synapse, 62, 243-252.

Garris, P.A. \& Wightman, R.M. (1994) Different kinetics govern dopaminergic transmission in the amygdala, prefrontal cortex, and striatum: an in vivo voltammetric study. J. Neurosci., 14, 442-450.

Gysling, K. \& Wang, R.Y. (1983) Morphine-induced activation of A10 dopamine neurons in the rat. Brain Res., 277, 119-127.

Heien, M.L., Khan, A.S., Ariansen, J.L., Cheer, J.F., Phillips, P.E., Wassum, K.M. \& Wightman, R.M. (2005) Real-time measurement of dopamine fluctuations after cocaine in the brain of behaving rats. Proc. Natl. Acad. Sci. USA, 102, 10023-10028.

Hyman, S.E., Malenka, R.C. \& Nestler, E.J. (2006) Neural mechanisms of addiction: the role of reward-related learning and memory. Annu. Rev. Neurosci., 29, 565-598.

Ikemoto, S. (2007) Dopamine reward circuitry: two projection systems from the ventral midbrain to the nucleus accumbens-olfactory tubercle complex. Brain Res. Rev., 56, 27-78.

Ito, R., Dalley, J.W., Howes, S.R., Robbins, T.W. \& Everitt, B.J. (2000) Dissociation in conditioned dopamine release in the nucleus accumbens core and shell in response to cocaine cues and during cocaine-seeking behavior in rats. J. Neurosci., 20, 7489-7495.

Johnson, S.W. \& North, R.A. (1992) Opioids excite dopamine neurons by hyperpolarization of local interneurons. J. Neurosci., 12, 483-488.

Kalso, E. (2007) How different is oxycodone from morphine? Pain, 132, $227-228$.

Keithley, R.B. \& Wightman, R.M. (2011) Assessing principal component regression prediction of neurochemicals detected with fast-scan cyclic voltammetry. ACS Chem. Neurosci., 2, 514-525.

Keithley, R.B., Heien, M.L. \& Wightman, R.M. (2009) Multivariate concentration determination using principal component regression with residual analysis. TRAC-Trend. Anal. Chem., 28, 1127-1136.

Keithley, R.B., Carelli, R.M. \& Wightman, R.M. (2010) Rank estimation and the multivariate analysis of in vivo fast-scan cyclic voltammetric data. Anal. Chem., 82, 5541-5551.

Kelley, A.E. (2004) Ventral striatal control of appetitive motivation: role in ingestive behavior and reward-related learning. Neurosci. Biobehav. R., 27, 765-776.

Lalovic, B., Kharasch, E., Hoffer, C., Risler, L., Liu-Chen, L.-Y. \& Shen, D.D. (2006) Pharmacokinetics and pharmacodynamics of oral oxycodone in healthy human subjects: role of circulating active metabolites*. Clin. Pharmacol. Ther., 79, 461-479.

Lammel, S., Hetzel, A., Hackel, O., Jones, I., Liss, B. \& Roeper, J. (2008) Unique properties of mesoprefrontal neurons within a dual mesocorticolimbic dopamine system. Neuron, 57, 760-773.

Lammel, S., Ion, D.I., Roeper, J. \& Malenka, R.C. (2011) Projection-specific modulation of dopamine neuron synapses by aversive and rewarding stimuli. Neuron, 70, 855-862.

Lemberg, K.K., Heiskanen, T.E., Kontinen, V.K. \& Kalso, E.A. (2009) Pharmacology of oxycodone: does it explain why oxycodone has become a bestselling strong opioid? Scand. J. Pain, 1, S18-S23.

Lester, P.A. \& Traynor, J.R. (2006) Comparison of the in vitro efficacy of $\mu$, $\delta, \kappa$ and $\mathrm{ORL}_{1}$ receptor agonists and non-selective opioid agonists in dog brain membranes. Brain Res., 1073, 290-296.

Lüscher, C. \& Ungless, M.A. (2006) The mechanistic classification of addictive drugs. PLoS Med., 3, e437.

Matsui, A. \& Williams, J.T. (2011) Opioid-sensitive GABA inputs from rostromedial tegmental nucleus synapse onto midbrain dopamine neurons. $J$. Neurosci., 31, 17729-17735.

Matthews, R.T. \& German, D.C. (1984) Electrophysiological evidence for excitation of rat ventral tegmental area dopamine neurons by morphine Neuroscience, 11, 617-625.

Mayer-Blackwell, B., Schlussman, S.D., Butelman, E.R., Ho, A., Ott, J., Kreek, M.J. \& Zhang, Y. (2014) Self administration of oxycodone by adolescent and adult mice affects striatal neurotransmitter receptor gene expression. Neuroscience, 258, 280-291. 
McCabe, S.E., West, B.T. \& Boyd, C.J. (2013a) Leftover prescription opioids and nonmedical use among high school seniors: a multi-cohort national study. J. Adolescent Health, 52, 480-485.

McCabe, S.E., West, B.T. \& Boyd, C.J. (2013b) Medical use, medical misuse, and nonmedical use of prescription opioids: results from a longitudinal study. Pain, 154, 708-713.

Melief, E.J., Miyatake, M., Bruchas, M.R. \& Chavkin, C. (2010) Liganddirected c-Jun N-terminal kinase activation disrupts opioid receptor signaling. Proc. Natl. Acad. Sci. USA, 107, 11608-11613.

Michael, D.J., Joseph, J.D., Kilpatrick, M.R., Travis, E.R. \& Wightman, R.M. (1999) Improving data acquisition for fast-scan cyclic voltammetry. Anal. Chem., 71, 3941-3947.

Mierzejewski, P., Koros, E., Goldberg, S.R., Kostowski, W. \& Stefanski, R. (2003) Intravenous self-administration of morphine and cocaine: a comparative study. Pol. J. Pharmacol., 55, 713-726.

Nestler, E.J. \& Malenka, R.C. (2004) The addicted brain. Sci. Am., 290, 78-85.

Nielsen, C.K., Ross, F.B., Lotfipour, S., Saini, K.S., Edwards, S.R. \& Smith, M.T. (2007) Oxycodone and morphine have distinctly different pharmacological profiles: radioligand binding and behavioural studies in two rat models of neuropathic pain. Pain, 132, 289-300.

Niikura, K., Ho, A., Kreek, M.J. \& Zhang, Y. (2013) Oxycodone-induced conditioned place preference and sensitization of locomotor activity in adolescent and adult mice. Pharmacol. Biochem. Be., 110, 112-116.

Owesson-White, C.A., Roitman, M.F., Sombers, L.A., Belle, A.M., Keithley, R.B., Peele, J.L., Carelli, R.M. \& Wightman, R.M. (2012) Sources contributing to the average extracellular concentration of dopamine in the nucleus accumbens. J. Neurochem., 121, 252-262.

Parker, L.A., Cyr, J.A., Santi, A.N. \& Burton, P.D. (2002) The aversive properties of acute morphine dependence persist $48 \mathrm{~h}$ after a single exposure to morphine: evaluation by taste and place conditioning. Pharmacol. Biochem. Be., 72, 87-92.

Peckham, E.M. \& Traynor, J.R. (2006) Comparison of the antinociceptive response to morphine and morphine-like compounds in male and female Sprague-Dawley rats. J. Pharmacol. Exp. Ther., 316, 1195-1201.

Phillips, P.E., Robinson, D.L., Stuber, G.D., Carelli, R.M. \& Wightman, R.M. (2003a) Real-time measurements of phasic changes in extracellular dopamine concentration in freely moving rats by fast-scan cyclic voltammetry. Method. Mol. Med., 79, 443-464.

Phillips, P.E., Stuber, G.D., Heien, M.L., Wightman, R.M. \& Carelli, R.M. (2003b) Subsecond dopamine release promotes cocaine seeking. Nature, 422, 614-618.

Pontieri, F.E., Tanda, G. \& Di Chiara, G. (1995) Intravenous cocaine, morphine, and amphetamine preferentially increase extracellular dopamine in the "shell" as compared with the "core" of the rat nucleus accumbens. Proc. Natl. Acad. Sci. USA, 92, 12304-12308.

Porter-Stransky, K.A., Wescott, S.A., Hershman, M., Badrinarayan, A., Vander Weele, C.M., Lovic, V. \& Aragona, B.J. (2011) Cocaine must enter the brain to evoke unconditioned dopamine release within the nucleus accumbens shell. Neurosci. Lett., 504, 13-17.

Robinson, T.E. \& Berridge, K.C. (2003) Addiction. Annu. Rev. Psychol., 54, $25-53$.

Robinson, D.L., Venton, B.J., Heien, M.L. \& Wightman, R.M. (2003) Detecting subsecond dopamine release with fast-scan cyclic voltammetry in vivo. Clin. Chem., 49, 1763-1773.

Roitman, M.F., Stuber, G.D., Phillips, P.E., Wightman, R.M. \& Carelli, R.M. (2004) Dopamine operates as a subsecond modulator of food seeking. J. Neurosci., 24, 1265-1271.

Rouge-Pont, F., Usiello, A., Benoit-Marand, M., Gonon, F., Piazza, P.V. \& Borrelli, E. (2002) Changes in extracellular dopamine induced by morphine and cocaine: crucial control by D2 receptors. J. Neurosci., 22, 3293-3301.

Saigusa, T., Aono, Y., Mizoguchi, N., Iwakami, T., Takada, K., Oi, Y., Ueda, K., Koshikawa, N. \& Cools, A.R. (2008) Role of GABA B $_{\text {receptors }}$ in the endomorphin-1-, but not endomorphin-2-, induced dopamine efflux in the nucleus accumbens of freely moving rats. Eur. J. Pharmacol., 581, 276-282.
Shen, H.W., Scofield, M.D., Boger, H., Hensley, M. \& Kalivas, P.W. (2014) Synaptic glutamate spillover due to impaired glutamate uptake mediates heroin relapse. J. Neurosci., 34, 5649-5657.

Sinkala, E., McCutcheon, J.E., Schuck, M.J., Schmidt, E., Roitman, M.F. \& Eddington, D.T. (2012) Electrode calibration with a microfluidic flow cell for fast-scan cyclic voltammetry. Lab Chip, 12, 2403-2408.

Sombers, L.A., Beyene, M., Carelli, R.M. \& Wightman, R.M. (2009) Synaptic overflow of dopamine in the nucleus accumbens arises from neuronal activity in the ventral tegmental area. J. Neurosci., 29, 1735-1742.

Song, P., Mabrouk, O.S., Hershey, N.D. \& Kennedy, R.T. (2012) In vivo neurochemical monitoring using benzoyl chloride derivatization and liquid chromatography-mass spectrometry. Anal. Chem., 84, 412-419.

Stoops, W.W., Hatton, K.W., Lofwall, M.R., Nuzzo, P.A. \& Walsh, S.L. (2010) Intravenous oxycodone, hydrocodone, and morphine in recreational opioid users: abuse potential and relative potencies. Psychopharmacology, 212, 193-203.

Sun, J.Y., Yang, J.Y., Wang, F., Wang, J.Y., Song, W., Su, G.Y., Dong, Y.X. \& Wu, C.F. (2011) Lesions of nucleus accumbens affect morphineinduced release of ascorbic acid and GABA but not of glutamate in rats. Addict. Biol., 16, 540-550.

Takmakov, P., Zachek, M.K., Keithley, R.B., Bucher, E.S., McCarty, G.S. \& Wightman, R.M. (2010) Characterization of local $\mathrm{pH}$ changes in brain using fast-scan cyclic voltammetry with carbon microelectrodes. Anal. Chem., 82, 9892-9900.

Tan, K.R., Yvon, C., Turiault, M., Mirzabekov, J.J., Doehner, J., Labouèbe, G., Deisseroth, K., Tye, K.M. \& Lüscher, C. (2012) GABA neurons of the VTA drive conditioned place aversion. Neuron, 73, 1173-1183.

Thompson, P.I., Joel, S.P., John, L., Wedzicha, J.A., Maclean, M. \& Slevin, M.L. (1995) Respiratory depression following morphine and morphine-6glucuronide in normal subjects. Brit. J. Clin. Pharmaco., 40, 145-152.

Vander Weele, C.M., Lovic, V. \& Aragona, B.J. (2011) Rapid dopamine signaling in response to a short-acting opiatae, remifentanil. Society for Neuroscience. 41st Annual Meeting. Washington, District of Columbia.

Venton, B.J., Michael, D.J. \& Wightman, R.M. (2003) Correlation of local changes in extracellular oxygen and $\mathrm{pH}$ that accompany dopaminergic terminal activity in the rat caudate-putamen. J. Neurochem., 84, 373-381.

Villesen, H.H., Foster, D.J., Upton, R.N., Somogyi, A.A., Martinez, A. \& Grant, C. (2006) Cerebral kinetics of oxycodone in conscious sheep. $J$. Pharm. Sci., 95, 1666-1676.

Wightman, R.M., Heien, M.L., Wassum, K.M., Sombers, L.A., Aragona, B.J., Khan, A.S., Ariansen, J.L., Cheer, J.F., Phillips, P.E. \& Carelli, R.M. (2007) Dopamine release is heterogeneous within microenvironments of the rat nucleus accumbens. Eur. J. Neurosci., 26, 2046-2054.

Will, M., Franzblau, E. \& Kelley, A. (2003) Nucleus accumbens $\mu$-opioids regulate intake of a high-fat diet via activation of a distributed brain network. J. Neurosci., 23, 2882-2888.

Williams, J.T., Christie, M.J. \& Manzoni, O. (2001) Cellular and synaptic adaptations mediating opioid dependence. Physiol. Rev., 81, 299-343.

Willuhn, I., Burgeno, L.M., Everitt, B.J. \& Phillips, P.E. (2012) Hierarchical recruitment of phasic dopamine signaling in the striatum during the progression of cocaine use. Proc. Natl. Acad. Sci. USA, 109, 20703-20708.

Wydra, K., Golembiowska, K., Zaniewska, M., Kamińska, K., Ferraro, L., Fuxe, K. \& Filip, M. (2013) Accumbal and pallidal dopamine, glutamate and GABA overflow during cocaine self-administration and its extinction in rats. Addict. Biol., 18, 307-324.

van Zessen, R., Phillips, J.L., Budygin, E.A. \& Stuber, G.D. (2012) Activation of VTA GABA neurons disrupts reward consumption. Neuron, 73, $1184-1194$.

Zhang, Y., Picetti, R., Butelman, E.R., Schlussman, S.D., Ho, A. \& Kreek, M.J. (2009) Behavioral and neurochemical changes induced by oxycodone differ between adolescent and adult mice. Neuropsychopharmacology, 34, 912-922.

Zhang, Y., Mayer-Blackwell, B., Schlussman, S.D., Randesi, M., Butelman, E.R., Ho, A., Ott, J. \& Kreek, M.J. (2014) Extended access oxycodone selfadministration and neurotransmitter receptor gene expression in the dorsal striatum of adult C57BL/6 J mice. Psychopharmacology, 231, 1277-1287. 